\title{
Convergence of Finite Element Approximations of Large Eddy Motion
}

\author{
Traian Iliescu, ${ }^{1}$ Volker John, ${ }^{2}$ William J. Layton ${ }^{3}$ \\ ${ }^{1}$ Mathematics and Computer Science Division, Argonne National Laboratory, \\ Argonne, Illinois 60439 \\ ${ }^{2}$ Faculty of Mathematics, Otto-von-Guericke-University, Magdeburg, Germany \\ ${ }^{3}$ Department of Mathematics, University of Pittsburgh, \\ Pittsburgh, Pennsylvania 15260
}

Received 21 March 2001; accepted 25 March 2002

Fluid motion in many applications occurs at higher Reynolds numbers. In these applications dealing with turbulent flow is thus inescapable. One promising approach to the simulation of the motion of the large structures in turbulent flow is large eddy simulation in which equations describing the motion of local spatial averages of the fluid velocity are solved numerically. This report considers "numerical errors" in LES. Specifically, for one family of space filtered flow models, we show convergence of the finite element approximation of the model and give an estimate of the error. ๑ 2002 Wiley Periodicals, Inc. Numer Methods Partial Differential Eq 18: 689-710, 2002; Published online in Wiley InterScience (www.interscience.wiley.com); DOI 10.1002/num. 10027

Keywords: Navier-Stokes equations; large eddy simulation; finite element method

\section{INTRODUCTION}

Consider the (turbulent) flow of an incompressible fluid. One promising and common approach to the simulation of the motion of the large fluid structures is large eddy simulation (LES). Various models are used in LES; a common one is to find $(\mathbf{w}, q)$, where $\mathbf{w}: \Omega\left(\subset \mathbb{R}^{d}, d=2\right.$ or 3 , polygonal domain) $\times[0, T] \rightarrow \mathbb{R}^{d}, q: \Omega \times(0, T] \rightarrow \mathbb{R}$ satisfying

$$
\begin{aligned}
\mathbf{w}_{t}+\mathbf{w} \cdot \nabla \mathbf{w}-R e^{-1} \Delta \mathbf{w}+\nabla q-\nabla \cdot\left(\nu_{T}(\nabla \mathbf{w}) \nabla \mathbf{w}\right)+\mu_{1} \delta^{2} \nabla \cdot\left(A^{-1}(\nabla \mathbf{w} \nabla \mathbf{w})\right) & =\overline{\mathbf{f}} & \text { in } \Omega \times(0, T] \\
\nabla \cdot \mathbf{w} & =0 & \text { in } \Omega \times[0, T] \\
\mathbf{w}(\mathbf{x}, 0) & =\overline{\mathbf{u}}_{0}(\mathbf{x}) & \text { in } \Omega
\end{aligned}
$$$$
+ \text { boundary conditions on } \partial \Omega \text {. }
$$

Correspondence to: William J. Layton, Department of Mathematics, University of Pittsburgh, 301 Thackeray Hall, Pittsburgh, PA 15260 (e-mail: wj1@ pitt.edu)

(C) 2002 Wiley Periodicals, Inc. 
The notation and terms in (1.1) require some explanation. The operator $A^{-1}$ denotes a regularization operator, introduced in [1], which is described below. The term $\nabla \mathbf{w} \nabla \mathbf{w}$ is shorthand for the tensor

$$
(\nabla \mathbf{w} \nabla \mathbf{w})_{i j}:=\sum_{l=1}^{d} \frac{\partial \mathbf{w}_{i}}{\partial \mathbf{x}_{l}} \frac{\partial \mathbf{w}_{j}}{\partial \mathbf{x}_{l}}
$$

The function $\nu_{T}(\nabla \mathbf{w})$ is the "turbulent viscosity" coefficient arising from the subgrid scale model used for turbulent fluctuations. There are many mathematically interesting possibilities for $\nu_{T}(\nabla \mathbf{w})$; see [2-9]. In this article we study the most commonly used Smagorinsky [4] model

$$
\nu_{T}(\nabla \mathbf{w})=\mu_{0} \delta^{2}|\nabla \mathbf{w}|, \quad|\nabla \mathbf{w}|=\left(\sum_{i, j=1}^{d}\left(\frac{\partial w_{i}}{\partial x_{j}}\right)^{2}\right)^{1 / 2} .
$$

The parameter $\delta$ denotes the "averaging radius": the model (1.1) seeks to provide an approximation of the eddies of size $O(\delta)$ or larger. The data $\overline{\mathbf{f}}, \overline{\mathbf{u}}_{0}$ are $O(\delta)$ averages of data from some turbulent flow problem underlying (1.1). The domain $\Omega$ is assumed to be polyhedral and bounded with boundary $\Gamma$.

The question of boundary conditions for (1.1) is a fundamental question in LES. There are various proposals; we impose a boundary condition suggested in [1] and developed in [10]. If the fluid particles adhere to the walls, it does not follow that the large eddies also "stick." In fact, it is clear that large eddies do move slip along walls and lose energy as they slip. The conditions we impose are no penetration (1.3) and slip with resistance (1.4):

$$
\begin{aligned}
\mathbf{w} \cdot \hat{n} & =0, \quad \text { on } \Gamma, \\
\mathbf{w} \cdot \hat{\tau}_{j}+\beta^{-1}(\delta, \operatorname{Re}) \mathbf{t} \cdot \hat{\tau}_{j} & =0, \quad \text { and } \Gamma, j=1, d-1 .
\end{aligned}
$$

Here $\beta(\delta, R e)$ is the friction coefficient, and the vectors $\hat{n}$ and $\hat{\tau}_{j}$ (where $j=1$ if $d=2$ and $j=1,2$ if $d=3$ ) denote the unit normal and tangent vectors to $\Gamma$ where, if $d=3, \hat{\tau}_{1} \perp \hat{\tau}_{2}$. The computational choice of $\hat{n}$ and $\hat{\tau}_{j}$ at the corners in such boundary conditions is resolved in the work by Gresho and Sani [11]. If $d=3$, all terms in which $\hat{\tau}_{j}$ occurs should (by understanding) be summed from $j=1,2$; for example, $\left\|\mathbf{w} \cdot \hat{\tau}_{j}\right\|_{\Gamma}^{2}$ means $\sum_{j=1}^{2}\left\|\mathbf{w} \cdot \hat{\tau}_{j}\right\|_{\Gamma}^{2}$. Also $\mathbf{t}$ represents the Cauchy stress vector associated with w. Specifically,

$$
\mathbf{t}=\hat{n} \cdot\left[-q I-\mu_{1} \delta^{2} A^{-1}(\nabla \mathbf{w} \nabla \mathbf{w})+R e^{-1} \nabla \mathbf{w}+\nu_{t}(\nabla \mathbf{w}) \nabla \mathbf{w}\right]
$$

There are several natural choices for the regularization $A^{-1}$ in (1.1). The most commonly used model, which we consider here, is with no regularization $A^{-1} \equiv I$. This model evolved from the work of Leonard [12] and Clark, Ferziger, and Reynolds [13]. Its derivation is based on a Taylor polynomial approximation of the Fourier transform of the Gaussian filter wherefore it is called sometimes Taylor LES model (also known as the gradient or nonlinear or tensordiffusivity LES model). A systematic derivation can be found in Aldama [14], and it has been used for many computational studies, for example, Cantekin, Westerink, and Luettich [15] and Sagaut [9]. The report [1] suggests the inclusion of a regularization operator $A^{-1}$ in the system 
(1.1). One choice of $A^{-1}$ is simply to reapply the spatial filter underlying (1.1): $A^{-1} \mathbf{v}=g_{\delta} * \mathbf{v}$; another possibility is $A^{-1} \mathbf{v}=\left(-\delta^{2} \Delta+I\right)^{-1} \mathbf{v}$.

Large eddy simulation involves two fundamental issues: assessment of "modeling errors" and "numerical errors." Modeling errors refer to the question of how close $\mathbf{w}(\mathbf{x}, t)$ is to the true flow averages: $\|\mathbf{w}-\overline{\mathbf{u}}\|$ for some norm $\|\cdot\| \|$. To our knowledge, there are no analytical results to date on this question for (1.1), but there are experimental results comparing various averages of $\mathbf{w}$ to those same averages of $\overline{\mathbf{u}}$ (i.e., averages of averages of $\mathbf{u})$. If we accept $\mathbf{w}(\mathbf{x}, t)$ as an interesting model for $\overline{\mathbf{u}}$, numerical errors describe how close an approximation $\mathbf{w}^{h}$ is to $\mathbf{w}$. Numerical errors raise classical questions of stability, consistency, and convergence for approximations of (1.1).

This report considers precisely this question for finite element approximations of (1.1). In Theorem 3.4 we show that the usual, continuous-in-time, finite element approximation to (1.1), $\mathbf{w}^{h}$, converges to $\mathbf{w}$ as the mesh width $h \rightarrow 0$ for the Reynolds number $R e$ and averaging radius $\delta$ fixed.

This analysis leads to interesting questions beyond the case of the usual Navier-Stokes equations (pioneered by Heywood and Rannacher in a series of papers [16, 17]), including the case of slip with friction boundary conditions (1.4) (see, for example, [18, 19] for some work related to this case); the degeneracy of the $\mu$-Laplacian-based subgrid-scale model in (1.1) (see, for example, $[20,5]$ for numerical analysis of the equilibrium model composed of NSE + $\mu$-Laplacian); the "cross-term" $\delta^{2} \nabla \cdot(\nabla \mathbf{w} \nabla \mathbf{w})$ in (1.1), which is nonmonotone, nonlinear, and higher order; and the dependence of the error on the Reynolds number, $R e$, and the averaging radius, $\delta$.

Our convergence analysis comes to grips with some of these questions. In particular, we prove convergence as $h \rightarrow 0$ for fixed $R e$. In some sense, Theorem 3.4 shows that the parameter $\delta$ does not degrade convergence. Naturally, we expect that a sharper analysis will show that its presence in the model improves the estimates. The degeneracy in the Smagorinsky [4] subgridscale model is not an essential difficulty; but (surprisingly) its polynomial growth, which must match that of the cross term to ensure boundedness of the kinetic energy in $\mathbf{w}$, seems to cause suboptimality in the resulting error estimates. This issue has recently been studied in a simplified setting in [21].

Nevertheless, convergence $\mathbf{w}^{h} \rightarrow \mathbf{w}$ as $h \rightarrow 0$ is proven. The long-term analytical goals in the numerical analysis of large eddy simulation are then to extend the error analysis to the model, including the regularization operator $A^{-1}$; to sharpen this result, especially with respect to error dependence on $\delta$ and $R e$, where possible; and to complement it with analysis of the modeling error. Preliminary steps in this last direction have recently been taken in [22] for a different class of LES models.

\section{VARIATIONAL FORMULATION OF THE MODEL}

We now introduce the notation for the functional setting. The $L^{2}(\Omega)$ norm and inner product are denoted $\|\cdot\|$ and $(\cdot, \cdot)$. The $L^{2}(\Gamma)$ norm and inner product are denoted $\|\cdot\|_{\Gamma}$ and $((\cdot),(\cdot))_{\Gamma}$. The $L^{3}(\Omega)$ norm is $\|\cdot\|_{L^{3}}$, and the Sobolev $W^{k, p}(\Omega)$ norm is denoted $\|\cdot\|_{k, p}$, with $p$ omitted if $p=2$. See, for example, [23] for a clear development of Sobolev spaces focusing on those important for the Navier-Stokes equations. Generic constants independent of the mesh width $h$ are denoted by $C$.

It is natural to define the velocity-pressure spaces as follows: 


$$
\begin{aligned}
& X:=\left\{\mathbf{v} \in W^{1,3}(\Omega)^{d}: \mathbf{v} \cdot \hat{n}=0 \text { on } \Gamma,\right\} \\
& Q:=L_{0}^{2}(\Omega)=\left\{\lambda(\mathbf{x}) \in L^{2}(\Omega):(\lambda, 1)=0\right\}
\end{aligned}
$$

The Smagorinsky model used needs the assumption $\nabla \mathbf{v} \in L^{3}(\Omega)$.

Some existence results for weak solutions of (1.1)-(1.4) have recently been proven in [24-26]. The regularity of weak solutions to (1.1)-(1.4) is still an open question, including regularity down to $t=0$. We shall nevertheless assume that (1.1)-(1.4) has a unique solution in the sense of the variational formulation (2.1). We will attempt to minimize any additional regularity assumed, and it will be explicitly stated as it is used. Because the boundary conditions on $\mathbf{w}$ are not simple Dirichlet conditions, care must be taken in developing a variational formulation of $(1.1)$ in $(X, Q)$.

Consider the following term, for $\mathbf{v} \in X$ and $\mathbf{w}$ smooth enough:

$$
\begin{aligned}
& \int_{\Omega} \nabla \cdot\left[q I+\mu_{1} \delta^{2}(\nabla \mathbf{w} \nabla \mathbf{w})-\left(R e^{-1}+\nu_{T}(\nabla \mathbf{w})\right) \nabla \mathbf{w}\right] \cdot \mathbf{v} d \mathbf{x}=\int_{\Gamma} \hat{n} \cdot\left[q I+\mu_{1} \delta^{2}(\nabla \mathbf{w} \nabla \mathbf{w})\right. \\
& \left.-\left(R e^{-1}+\nu_{T}(\nabla \mathbf{w})\right) \nabla \mathbf{w}\right] \cdot \mathbf{v} d s-\int_{\Omega} q \nabla \cdot \mathbf{v}+\left[\mu_{1} \delta^{2}(\nabla \mathbf{w} \nabla \mathbf{w})-\left(R e^{-1} \nabla \mathbf{w}+\nu_{T}(\nabla \mathbf{w})\right) \nabla \mathbf{w}\right]: \nabla \mathbf{v} d \mathbf{x} .
\end{aligned}
$$

Decomposing $\mathbf{v}=\left(\mathbf{v} \cdot \hat{\tau}_{j}\right) \hat{\tau}_{j}+(\mathbf{v} \cdot \hat{n}) \hat{n}=\left(\mathbf{v} \cdot \hat{\tau}_{j}\right) \hat{\tau}_{j}$ in the first integral, canceling the obvious terms, and using (1.3)-(1.4), we obtain

$$
\begin{aligned}
\int_{\Omega} \nabla \cdot\left[q I+\mu_{1} \delta^{2}(\nabla \mathbf{w} \nabla \mathbf{w})-\left(R e^{-1}\right.\right. & \left.\left.+\nu_{T}(\nabla \mathbf{w})\right) \nabla \mathbf{w}\right] \cdot \mathbf{v} d \mathbf{x}=\beta(\delta, R e) \int_{\Gamma}\left(\mathbf{w} \cdot \hat{\tau}_{j}\right)\left(\mathbf{v} \cdot \hat{\tau}_{j}\right) d s \\
& -\int_{\Omega} q \nabla \cdot \mathbf{v}+\left[\mu_{1} \delta^{2}(\nabla \mathbf{w} \nabla \mathbf{w})-\left(R e^{-1}+\nu_{T}(\nabla \mathbf{w})\right) \nabla \mathbf{w}\right]: \nabla \mathbf{v} d \mathbf{x} .
\end{aligned}
$$

The next lemma is fundamental to energy estimation. Its proof is the same index calculation as in the case of the no-slip boundary condition.

Lemma 2.1. For any $\mathbf{u}, \mathbf{v}, \mathbf{w} \in X$ satisfying $\nabla \cdot \mathbf{v}=0$

$$
(\mathbf{v} \cdot \nabla \mathbf{w}, \mathbf{u})=-(\mathbf{v} \cdot \nabla \mathbf{u}, \mathbf{w})
$$

Thus,

$$
(\mathbf{v} \cdot \nabla \mathbf{w}, \mathbf{u})=\frac{1}{2}(\mathbf{v} \cdot \nabla \mathbf{w}, \mathbf{u})-\frac{1}{2}(\mathbf{v} \cdot \nabla \mathbf{u}, \mathbf{w})
$$

Proof. This follows since $\mathbf{v} \cdot \hat{n}=0$ on $\Gamma$ for all $\mathbf{v} \in X$ and $\nabla \cdot \mathbf{v}=0$.

We consider the skew-symmetric form of the convective term, which is denoted by

$$
b(\mathbf{u}, \mathbf{v}, \mathbf{w})=\frac{1}{2}((\mathbf{u} \cdot \nabla \mathbf{v}, \mathbf{w})-(\mathbf{u} \cdot \nabla \mathbf{w}, \mathbf{v}))
$$


A variational formulation of (1.1)-(1.4) is thus as follows. For $\overline{\mathbf{f}} \in L^{2}\left(0, T ; L^{2}(\Omega)\right)$, find a differentiable map w $:[0, T] \rightarrow X, q:(0, T] \rightarrow Q$ satisfying

$$
\begin{aligned}
\left(\mathbf{w}_{t}, \mathbf{v}\right)+\left(\left(\operatorname{Re}^{-1}+\right.\right. & \left.\left.\nu_{T}(\nabla \mathbf{w})\right) \nabla \mathbf{w}, \nabla \mathbf{v}\right)+\beta(\delta, R e)\left(\mathbf{w} \cdot \hat{\tau}_{j}, \mathbf{v} \cdot \hat{\tau}_{j}\right)_{\Gamma} \\
& -(q, \nabla \cdot \mathbf{v})+b(\mathbf{w}, \mathbf{w}, \mathbf{v})-\mu_{1} \delta^{2}((\nabla \mathbf{w} \nabla \mathbf{w}), \nabla \mathbf{v})=(\overline{\mathbf{f}}, \mathbf{v}),(\lambda, \nabla \cdot \mathbf{w})=0
\end{aligned}
$$

for all $(\mathbf{v}, \lambda) \in(X, Q)$.

We will frequently use the Poincaré inequality for all $\mathbf{v} \in X$

$$
\|\mathbf{v}\| \leq C\|\nabla \mathbf{v}\|
$$

Note that this inequality needs only $\mathbf{v} \cdot \hat{n}=0$ on $\Gamma$ to hold, rather than $\mathbf{v}=\mathbf{0}$ on $\Gamma$; see Galdi [23], Section II.4.

The next two technical lemmas quantify the control the model of turbulent diffusion exerts over the interaction of large and small eddies. They are also the key for proving existence of weak solutions; see Coletti [25, 24] and Berselli et al. [26]. Define, for brevity,

$$
F(\mathbf{w}):=\left(R e^{-1}+\nu_{T}(\nabla \mathbf{w})\right) \nabla \mathbf{w}-\mu_{1} \delta^{2}(\nabla \mathbf{w} \nabla \mathbf{w})
$$

Lemma 2.2. Let $\nu_{T}(\nabla \mathbf{w}):=\mu_{0} \delta^{2}|\nabla \mathbf{w}|$, where $\mu_{0} \geq 4 \mu_{1}$. Then, there is a constant $\underline{C}$ such that for any $\mathbf{v}_{1}, \mathbf{v}_{2} \in X$,

$$
\begin{aligned}
& \left(F\left(\mathbf{v}_{1}\right)-F\left(\mathbf{v}_{2}\right), \nabla\left(\mathbf{v}_{1}-\mathbf{v}_{2}\right)\right)+\beta(\delta, R e)\left(\left(\mathbf{v}_{1}-\mathbf{v}_{2}\right) \cdot \hat{\tau}_{j},\left(\mathbf{v}_{1}-\mathbf{v}_{2}\right) \cdot \hat{\tau}_{j}\right)_{\Gamma} \\
& \quad \geq \operatorname{Re}^{-1}\left\|\nabla\left(\mathbf{v}_{1}-\mathbf{v}_{2}\right)\right\|^{2}+\frac{\underline{C}}{2} \mu_{0} \delta^{2}\left\|\nabla\left(\mathbf{v}_{1}-\mathbf{v}_{2}\right)\right\|_{L^{3}}^{3}+\beta(\delta, R e)\left\|\left(\mathbf{v}_{1}-\mathbf{v}_{2}\right) \cdot \hat{\tau}_{j}\right\|_{\Gamma}^{2} .
\end{aligned}
$$

Proof. The proof of a similar estimate can be found in Coletti [25], which in turn is based on a proof by Ladyzhenskaya [27]. Because we need an explicit relation between $\mu_{0}$ and $\mu_{1}$ in our new setting, we will present here the proof to highlight the condition $\mu_{0} \geq 4 \mu_{1}$.

Let

$$
\tilde{F}(\mathbf{w}):=\left(R e^{-1}+\frac{1}{2} \mu_{0} \delta^{2}|\nabla \mathbf{w}|\right) \nabla \mathbf{w}-\mu_{1} \delta^{2}(\nabla \mathbf{w} \nabla \mathbf{w})
$$

Note that $F(\mathbf{w})=\tilde{F}(\mathbf{w})+\frac{1}{2} \mu_{0} \delta^{2}|\nabla \mathbf{w}| \nabla \mathbf{w}$.

Letting $\mathbf{v}^{\gamma}:=\gamma \mathbf{v}_{1}+(1-\gamma) \mathbf{v}_{2}, \gamma \in[0,1]$, and using the $\mu$-Laplacian's strong monotonicity (see, for example, [5]) and the approach in [25], we get

$$
\begin{aligned}
& \left(F\left(\mathbf{v}_{1}\right)-F\left(\mathbf{v}_{2}\right), \nabla\left(\mathbf{v}_{1}-\mathbf{v}_{2}\right)\right)+\beta(\delta, R e)\left(\left(\mathbf{v}_{1}-\mathbf{v}_{2}\right) \cdot \hat{\tau}_{j},\left(\mathbf{v}_{1}-\mathbf{v}_{2}\right) \cdot \hat{\tau}_{j}\right)_{\Gamma} \\
& =\beta(\delta, R e)\left\|\left(\mathbf{v}_{1}-\mathbf{v}_{2}\right) \cdot \hat{\tau}_{j}\right\|_{\Gamma}^{2}+\left(F\left(\mathbf{v}_{1}\right)-F\left(\mathbf{v}_{2}\right), \nabla\left(\mathbf{v}_{1}-\mathbf{v}_{2}\right)\right) \\
& \geq \beta(\delta, R e)\left\|\left(\mathbf{v}_{1}-\mathbf{v}_{2}\right) \cdot \hat{\tau}_{j}\right\|_{\Gamma}^{2}+\int_{\Omega}\left(\int_{0}^{1} \frac{d}{d \gamma} \tilde{F}\left(\mathbf{v}^{\gamma}\right) d \gamma\right) \nabla\left(\mathbf{v}_{1}-\mathbf{v}_{2}\right) d x \\
& \quad+\frac{C}{2} \mu_{0} \delta^{2}\left\|\nabla\left(\mathbf{v}_{1}-\mathbf{v}_{2}\right)\right\|_{L^{3}}^{3} .
\end{aligned}
$$


We now start to evaluate the second term on the right-hand side of the last inequality. In the sequel, by convention, repeated indices denote summation.

$$
\begin{aligned}
\frac{d}{d \gamma} \tilde{F}\left(\mathbf{v}^{\gamma}\right) \nabla\left(\mathbf{v}_{1}-\mathbf{v}_{2}\right)= & \left.R e^{-1} \frac{d}{d \gamma} \nabla \mathbf{v}^{\gamma}+\frac{1}{2} \mu_{0} \delta^{2} \frac{d}{d \gamma}\left(\left|\nabla \mathbf{v}^{\gamma}\right| \nabla \mathbf{v}^{\gamma}\right)-\mu_{1} \delta^{2} \frac{d}{d \gamma}\left(\nabla \mathbf{v}^{\gamma} \nabla \mathbf{v}^{\gamma}\right)\right] \\
& \times \nabla\left(\mathbf{v}_{1}-\mathbf{v}_{2}\right)=R e^{-1} \frac{d}{d \gamma} \frac{\partial \mathbf{v}_{i}^{\gamma}}{\partial \mathbf{x}_{j}}\left(\frac{\partial \mathbf{v}_{1 i}}{\partial \mathbf{x}_{j}}-\frac{\partial \mathbf{v}_{2 i}}{\partial \mathbf{x}_{j}}\right)+\frac{1}{2} \mu_{0} \delta^{2} \frac{d}{d \gamma} \\
& \times\left[\left(\frac{\sum}{k, l}\left(\frac{\partial \mathbf{v}_{l}^{\gamma}}{\partial \mathbf{x}_{k}}\right)^{2}\right)^{1 / 2} \frac{\partial \mathbf{v}_{i}^{\gamma}}{\partial \mathbf{x}_{j}}\right]\left(\frac{\partial \mathbf{v}_{1 i}}{\partial \mathbf{x}_{j}}-\frac{\partial \mathbf{v}_{2 i}}{\partial \mathbf{x}_{j}}\right)^{-} \mu_{1} \delta^{2} \frac{d}{d \gamma}\left[\frac{\partial \mathbf{v}_{i}^{\gamma}}{\partial \mathbf{x}_{l}} \frac{\partial \mathbf{v}_{j}^{\gamma}}{\partial \mathbf{x}_{l}}\right]\left(\frac{\partial \mathbf{v}_{1 i}}{\partial \mathbf{x}_{j}}-\frac{\partial \mathbf{v}_{2 i}}{\partial \mathbf{x}_{j}}\right) \\
= & R e^{-1}\left(\frac{\partial \mathbf{v}_{1 i}}{\partial \mathbf{x}_{j}}-\frac{\partial \mathbf{v}_{2 i}}{\partial \mathbf{x}_{j}}\right)^{2}+\frac{1}{2} \mu_{0} \delta^{2}\left[\sum\left(\frac{\partial \mathbf{v}_{l}^{\gamma}}{\partial \mathbf{x}_{k}}\right)^{2}\right]^{-1 / 2}\left[\left(\frac{\partial \mathbf{v}_{l}^{\gamma}}{\partial \mathbf{x}_{k}}\right)\left(\frac{\partial \mathbf{v}_{1 l}}{\partial \mathbf{x}_{k}}-\frac{\partial \mathbf{v}_{2 l}}{\partial \mathbf{x}_{k}}\right)\right] \\
& \left.\times\left[\left(\frac{\partial \mathbf{v}_{i}^{\gamma}}{\partial \mathbf{x}_{j}}\right)\left(\frac{\partial \mathbf{v}_{1 i}}{\partial \mathbf{x}_{j}}-\frac{\partial \mathbf{v}_{2 i}}{\partial \mathbf{x}_{j}}\right)\right]+\frac{1}{2} \mu_{0} \delta^{2}\left[\sum \frac{\partial \mathbf{v}_{l}^{\gamma}}{k, l}\right)^{2}\right]^{1 / 2}\left(\frac{\partial \mathbf{v}_{1 i}}{\partial \mathbf{x}_{j}}-\frac{\partial \mathbf{v}_{2 i}}{\partial \mathbf{x}_{j}}\right) \\
& \times\left(\frac{\partial \mathbf{v}_{1 i}}{\partial \mathbf{x}_{j}}-\frac{\partial \mathbf{v}_{2 i}}{\partial \mathbf{x}_{j}}\right)-\mu_{1} \delta^{2}\left[\left(\frac{\partial \mathbf{v}_{1 i}}{\partial \mathbf{x}_{l}}-\frac{\partial \mathbf{v}_{2 i}}{\partial \mathbf{x}_{l}}\right)^{2}\right) \\
& \left.\times \frac{\partial \mathbf{v}_{j}^{\gamma}}{\partial \mathbf{x}_{l}}+\frac{\partial \mathbf{v}_{i}^{\gamma}}{\partial \mathbf{x}_{l}}\left(\frac{\partial \mathbf{v}_{1 j}}{\partial \mathbf{x}_{l}}-\frac{\partial \mathbf{v}_{2 j}}{\partial \mathbf{x}_{l}}\right)\right]\left(\frac{\partial \mathbf{v}_{1 i}}{\partial \mathbf{x}_{j}}-\frac{\partial \mathbf{v}_{2 i}}{\partial \mathbf{x}_{j}}\right) .
\end{aligned}
$$

In the last equality, dropping the second term (which is positive) and using (twice) the Cauchy-Schwarz inequality for the last term, we get for $\mu_{0} \geq 4 \mu_{1}$

$$
\begin{aligned}
\frac{d}{d \gamma} \tilde{F}\left(\mathbf{v}^{\gamma}\right) \nabla\left(\mathbf{v}_{1}-\mathbf{v}_{2}\right) \geq & R e^{-1}\left|\nabla\left(\mathbf{v}_{1}-\mathbf{v}_{2}\right)\right|^{2}+\frac{1}{2} \mu_{0} \delta^{2}\left|\nabla \mathbf{v}^{\gamma}\right|\left|\nabla\left(\mathbf{v}_{1}-\mathbf{v}_{2}\right)\right|^{2} \\
& \quad-\mu_{1} \delta^{2}\left|\nabla \mathbf{v}^{\gamma}\right|\left|\nabla\left(\mathbf{v}_{1}-\mathbf{v}_{2}\right)\right|^{2}-\mu_{1} \delta^{2}\left|\nabla \mathbf{v}^{\gamma}\right|\left|\nabla\left(\mathbf{v}_{1}-\mathbf{v}_{2}\right)\right|^{2} \geq R e^{-1}\left|\nabla\left(\mathbf{v}_{1}-\mathbf{v}_{2}\right)\right|^{2}
\end{aligned}
$$

Inserting this estimate into (2.4) proves the statement of the lemma.

Remark: This lemma does not include the $\mathbf{w} \cdot \nabla \mathbf{w}$ nonlinearity describing how the large eddies convect themselves. Because of this $\mathbf{w} \cdot \nabla \mathbf{w}$ term, the nonlinearity in (1.1) is not monotonic.

The next technical lemma concerns the continuity properties of $F(\cdot)$.

Lemma 2.3. Assume $\nu_{T}(\nabla \mathbf{w}):=\mu_{0} \delta^{2}|\nabla \mathbf{w}|$. Then, there is a constant $\bar{C}$ such that for any $\mathbf{v}_{1}$, $\mathbf{v}_{2}, \phi \in X$ with $\left\|\nabla \mathbf{v}_{1}\right\|_{L^{3}} \leq r$ and $\left\|\nabla \mathbf{v}_{2}\right\|_{L^{3}} \leq r$,

$$
\left(F\left(\mathbf{v}_{1}\right)-F\left(\mathbf{v}_{2}\right), \nabla \phi\right) \leq \bar{C} r \delta^{2}\left\|\nabla\left(\mathbf{v}_{1}-\mathbf{v}_{2}\right)\right\|_{L^{3}}\|\nabla \phi\|_{L^{3}}+R e^{-1}\left\|\nabla\left(\mathbf{v}_{1}-\mathbf{v}_{2}\right)\right\|\|\nabla \phi\| .
$$

Proof. Using Hölder's inequality and adding and subtracting terms as appropriate, we get

$$
\begin{aligned}
\left(F\left(\mathbf{v}_{1}\right)-F\left(\mathbf{v}_{2}\right), \nabla \phi\right) \leq & R e^{-1}\left\|\nabla ( \mathbf { v } _ { 1 } - \mathbf { v } _ { 2 } ) \left|\|\mid \nabla \phi\|+\mu_{0} \delta^{2}\left(\left|\nabla \mathbf{v}_{1}\right| \nabla \mathbf{v}_{1}-\left|\nabla \mathbf{v}_{2}\right| \nabla \mathbf{v}_{2}, \nabla \phi\right)-\mu_{1} \delta^{2}\left(\nabla \mathbf{v}_{1} \nabla \mathbf{v}_{1}\right.\right.\right. \\
& \left.-\nabla \mathbf{v}_{2} \nabla \mathbf{v}_{2}, \nabla \phi\right) \leq R e^{-1}\left\|\nabla ( \mathbf { v } _ { 1 } - \mathbf { v } _ { 2 } ) \left|\|\mid \nabla \phi\|+\mu_{0} \delta^{2}\left(\left|\nabla \mathbf{v}_{1}\right| \nabla \mathbf{v}_{1}-\left|\nabla \mathbf{v}_{1}\right| \nabla \mathbf{v}_{2}\right.\right.\right.
\end{aligned}
$$




$$
\begin{aligned}
& \left.+\left|\nabla \mathbf{v}_{1}\right| \nabla \mathbf{v}_{2}-\left|\nabla \mathbf{v}_{2}\right| \nabla \mathbf{v}_{2}, \nabla \phi\right)-\mu_{1} \delta^{2}\left(\nabla \mathbf{v}_{1} \nabla \mathbf{v}_{1}-\nabla \mathbf{v}_{1} \nabla \mathbf{v}_{2}+\nabla \mathbf{v}_{1} \nabla \mathbf{v}_{2}\right. \\
& \left.-\nabla \mathbf{v}_{2} \nabla \mathbf{v}_{2}, \nabla \phi\right) \leq R e^{-1}\left\|\nabla\left(\mathbf{v}_{1}-\mathbf{v}_{2}\right)\right\|\|\nabla \phi\|+C \mu_{0} \delta^{2}\left\|\nabla \mathbf{v}_{1}\right\|_{L^{3}} \| \nabla\left(\mathbf{v}_{1}\right. \\
& \left.-\mathbf{v}_{2}\right)\left\|_{L^{3}}\right\| \nabla \phi\left\|_{L^{3}}+C \mu_{0} \delta^{2}\right\| \nabla\left(\mathbf{v}_{1}-\mathbf{v}_{2}\right)\left\|_{L^{3}}\right\| \nabla \mathbf{v}_{2}\|\|_{L^{3}}\|\nabla \phi\|_{L^{3}}+\mu_{1} \delta^{2}\left\|\nabla \mathbf{v}_{1}\right\|_{L^{3}} \| \nabla\left(\mathbf{v}_{1}\right. \\
& \left.-\mathbf{v}_{2}\right)\left\|_{L^{3}}\right\| \nabla \phi\left\|_{L^{3}}+\mu_{1} \delta^{2}\right\| \nabla\left(\mathbf{v}_{1}-\mathbf{v}_{2}\right)\left\|_{L^{3}}\right\| \nabla \mathbf{v}_{2}\left\|_{L^{3}}\right\| \nabla \phi \|_{L^{3}},
\end{aligned}
$$

which proves the lemma.

Using these lemmas, we can prove an energy bound for the solution of the continuous problem (1.1)-(1.4). This first bound is the foundation on which an existence theory for (1.1)-(1.4) is built.

Proposition 2.4. [Leray's inequality for the Large Eddy Model]. Let $\mathbf{w}(x, t)$ satisfy (2.1). Then, w satisfies the energy inequality

$$
\frac{1}{2}\|\mathbf{w}(t)\|^{2}+\int_{0}^{t}\left[\beta\left\|\mathbf{w} \cdot \hat{\tau}_{j}\right\|_{\Gamma}^{2}+R e^{-1}\|\nabla \mathbf{w}\|^{2}+\underline{C} \mu_{0} \delta^{2}\|\nabla \mathbf{w}\|_{L^{3}}^{3}\right] d t^{\prime} \leq \frac{1}{2}\|\mathbf{w}(0)\|^{2}+\int_{0}^{t}(\overline{\mathbf{f}}, \mathbf{w}) d t^{\prime},
$$

for any $t>0$. In particular,

$$
\|\nabla \mathbf{w}\|_{L^{3}\left(0, t, L^{3}\right)}^{3} \leq C\left(\delta, \mu_{0}\right)\left(\|\mathbf{w}(\mathbf{x}, 0)\|^{2}+\int_{0}^{t}\|\overline{\mathbf{f}}\|_{L^{1 / 5}}^{3 / 2}\right)=: C_{1}\left(\delta, \mu_{0}, t\right)
$$

Proof. Set $\mathbf{v}=\mathbf{w}$ and $\lambda=q$ in (2.1). Using Lemma 2.2 then gives

$$
\frac{1}{2} \frac{d}{d t}\|\mathbf{w}\|^{2}+R e^{-1}\|\nabla \mathbf{w}\|^{2}+C \underline{\mu_{0}} \delta^{2}\|\nabla \mathbf{w}\|_{L^{3}}^{3}+\beta\left\|\mathbf{w} \cdot \hat{\tau}_{j}\right\|_{\Gamma}^{2} \leq(\overline{\mathbf{f}}, \mathbf{w})
$$

from which the energy inequality follows by integration.

To prove the second part, use Hölder's inequality, the Sobolev embedding $W^{1,3}(\Omega) \rightarrow L^{6}(\Omega)$, Poincaré inequality (2.2), and Young's inequality to obtain

$$
(\overline{\mathbf{f}}, \mathbf{w}) \leq\|\mathbf{w}\|_{L^{\prime}}\|\overline{\mathbf{f}}\|_{L^{6 / 5}} \leq C\|\nabla \mathbf{w}\|_{L^{3}}\left\|||_{L^{6 / 5}} \leq \frac{\epsilon}{3}\right\| \nabla \mathbf{w}\left\|_{L^{3}}^{3}+\frac{C}{\epsilon^{1 / 2}}\right\| \overline{\mathbf{f}} \|_{L^{6 / 5}}^{3 / 2}
$$

Choosing $\epsilon=C \delta^{2}$ proves the second statement of Proposition 2.4.

Before proceeding with the error analysis of the nonlinear, time-dependent problem, we give estimates of two equilibrium projections. The first (Proposition 2.5) gives an estimate of the error in the nonlinear Galerkin projection obtained by dropping time dependence and convection (hence retaining only those terms associated with the turbulence modeling.) This estimate is not optimal, reflecting the quadratic growth in the model's nonlinearity. (Suboptimal estimates similar to this also occur in error analysis of problems such as the $\mu$-Laplacian [28], which are locally Lipschitz and strongly monotone in the sense of Vainberg [29].) Proposition 2.5 thus gives an idea of rates of convergence attainable in more complex settings as well. 
We assume that the velocity-pressure space $\left(X^{h}, Q^{h}\right)$ satisfies the natural $([18,19])$ inf-sup condition associated with slip with friction conditions on $\Gamma$. Note that since $\Gamma$ is polyhedral, the natural norm on $\Gamma$ is not the $H^{1 / 2}(\Gamma)$ norm but rather the sum of the $H^{1 / 2}\left(\Gamma_{i}\right)$ norms over all faces $\Gamma_{i}$ of $\Gamma$. Thus, we assume

$$
\inf _{\lambda^{h} \in Q^{h} \mathbf{v}^{h} \in X^{h}} \frac{\left(\lambda^{h}, \nabla \cdot \mathbf{v}^{h}\right)}{\left\|\lambda^{h}\right\|\left[\left\|\nabla \mathbf{v}^{h}\right\|^{2}+\sum_{\text {all faces } \Gamma_{i} \text { of } \Gamma}\left\|\mathbf{v}^{h} \cdot \hat{\tau}_{j}\right\|_{1 / 2, \Gamma_{i}}^{2}\right]^{1 / 2}} \geq \alpha>0 .
$$

Under this condition, the space of discretely divergence-free functions $V^{h}$

$$
V^{h}:=\left\{\mathbf{v}^{h} \in X^{h}:\left(\lambda^{h}, \nabla \cdot \mathbf{v}^{h}\right)=0, \quad \forall \lambda^{h} \in Q^{h}\right\}
$$

is not empty [30, 31]. Examples of finite element spaces satisfying (2.5) are constructed in [18, 19].

Proposition 2.5. Let $\chi^{h}$ denote an approximation of $\mathbf{w}$ in $V^{h}$ satisfying $\left\|\nabla \chi^{h}\right\|_{L^{3}} \leq C\|\nabla \mathbf{w}\|_{L^{3}}$. Assume also the conditions of Lemma 2.2 hold. Let $\tilde{\mathbf{w}} \in V^{h}$ be defined by

$$
\left(F(\mathbf{w})-F(\tilde{\mathbf{w}}), \nabla \mathbf{v}^{h}\right)+\beta\left((\mathbf{w}-\tilde{\mathbf{w}}) \cdot \hat{\tau}_{j}, \mathbf{v}^{h} \cdot \hat{\tau}_{j}\right)_{\Gamma}=0,
$$

for all $\mathbf{v}^{h} \in V^{h}$. Then, $\tilde{\mathbf{w}} \in V^{h}$ exists uniquely and the error $\mathbf{w}-\tilde{\mathbf{w}}$ satisfies

$$
\begin{aligned}
& \beta(\delta, \operatorname{Re})\left\|(\mathbf{w}-\tilde{\mathbf{w}}) \cdot \hat{\tau}_{j}\right\|_{\Gamma}^{2}+\operatorname{Re}^{-1}\|\nabla(\mathbf{w}-\tilde{\mathbf{w}})\|^{2}+\underline{C} \mu_{0} \delta^{2}\|\nabla(\mathbf{w}-\tilde{\mathbf{w}})\|_{L^{3}}^{3} \\
& \quad \leq C\left\{\left(\underline{C} \mu_{0}\right)^{-1 / 2}\left(\bar{C}\|\nabla \mathbf{w}\|_{L^{3}}\right)^{3 / 2} \delta^{2}\left\|\nabla\left(\mathbf{w}-\chi^{h}\right)\right\|_{L^{3}}^{3 / 2}+\operatorname{Re}^{-1}\left\|\nabla\left(\mathbf{w}-\chi^{h}\right)\right\|^{2}+\beta(\delta, R e)\left\|\left(\mathbf{w}-\chi^{h}\right) \cdot \hat{\tau}_{j}\right\|_{\Gamma}^{2}\right\} .
\end{aligned}
$$

Proof. That $\tilde{\mathbf{w}}$ exists uniquely follows from standard arguments using monotonicity following Minty's lemma, [32-34]. For the error estimate, adding and subtracting terms give

$\beta\left(\left(\chi^{h}-\tilde{\mathbf{w}}\right) \cdot \hat{\tau}_{j}, \mathbf{v}^{h} \cdot \hat{\tau}_{j}\right)_{\Gamma}+\left(F\left(\chi^{h}\right)-F(\tilde{\mathbf{w}}), \nabla \mathbf{v}^{h}\right)=\beta\left(\left(\chi^{h}-\mathbf{w}\right) \cdot \hat{\tau}_{j}, \mathbf{v}^{h} \cdot \tau_{j}\right)_{\Gamma}+\left(F\left(\chi^{h}\right)-F(\mathbf{w}), \nabla \mathbf{v}^{h}\right)$

for all $\mathbf{v}^{h} \in V^{h}$. Setting $\mathbf{v}^{h}=\chi^{h}-\tilde{\mathbf{w}}$ and using Lemma 2.2 gives

$$
\begin{aligned}
\beta\left\|\left(\chi^{h}-\tilde{\mathbf{w}}\right) \cdot \hat{\tau}_{j}\right\|_{\Gamma}^{2}+R e^{-1}\left\|\nabla\left(\chi^{h}-\tilde{\mathbf{w}}\right)\right\|^{2}+C \mu_{0} \delta^{2}\left\|\nabla\left(\chi^{h}-\tilde{\mathbf{w}}\right)\right\|_{L^{3}}^{3} \\
\quad \leq\left(F\left(\chi^{h}\right)-F(\mathbf{w}), \nabla\left(\chi^{h}-\tilde{\mathbf{w}}\right)\right)+\beta\left(\left(\chi^{h}-\mathbf{w}\right) \cdot \hat{\tau}_{j},\left(\chi^{h}-\tilde{\mathbf{w}}\right) \cdot \hat{\tau}_{j}\right)_{\Gamma} .
\end{aligned}
$$

Thus, using the Cauchy-Schwarz inequality, Young's inequality, and Lemma 2.3 give

$$
\begin{aligned}
& \beta\left\|\left(\chi^{h}-\tilde{\mathbf{w}}\right) \cdot \hat{\tau}_{j}\right\|_{\Gamma}^{2}+ R e^{-1}\left\|\nabla\left(\chi^{h}-\tilde{\mathbf{w}}\right)\right\|^{2}+C \underline{\mu_{0}} \delta^{2}\left\|\nabla\left(\chi^{h}-\tilde{\mathbf{w}}\right)\right\|_{L^{3}}^{3} \\
& \quad \leq \frac{1}{2} \operatorname{Re}^{-1}\left\|\nabla\left(\chi^{h}-\mathbf{w}\right)\right\|^{2}+\frac{1}{2} \operatorname{Re}^{-1}\left\|\nabla\left(\chi^{h}-\tilde{\mathbf{w}}\right)\right\|^{2} \\
&+\frac{\beta}{2}\left\|\left(\chi^{h}-\tilde{\mathbf{w}}\right) \cdot \hat{\tau}_{j}\right\|_{\Gamma}^{2}+\frac{\beta}{2}\left\|\left(\chi^{h}-\mathbf{w}\right) \cdot \hat{\tau}_{j}\right\|_{\Gamma}^{2}+\bar{C} r \delta^{2}\left\|\nabla\left(\chi^{h}-\tilde{\mathbf{w}}\right)\right\|_{L^{3}}\left\|\nabla\left(\mathbf{w}-\chi^{h}\right)\right\|_{L^{3}},
\end{aligned}
$$

where $r=\max \left\{\left\|\nabla \chi^{h}\right\|_{L^{3}},\|\nabla \mathbf{w}\|_{L^{3}}\right\}$, which is bounded by $C\|\nabla \mathbf{w}\|_{L^{3}}$. Collecting terms gives 


$$
\begin{aligned}
\beta\left\|\left(\chi^{h}-\tilde{\mathbf{w}}\right) \cdot \hat{\tau}_{j}\right\|_{\Gamma}^{2}+ & \operatorname{Re}^{-1}\left\|\nabla\left(\chi^{h}-\tilde{\mathbf{w}}\right)\right\|^{2}+2 \underline{C} \mu_{0} \delta^{2}\left\|\nabla\left(\chi^{h}-\tilde{\mathbf{w}}\right)\right\|_{L^{3}}^{3} \\
& \leq 2 \bar{C} r \delta^{2}\left\|\nabla\left(\chi^{h}-\tilde{\mathbf{w}}\right)\right\|_{L^{3}}\left\|\nabla\left(\mathbf{w}-\chi^{h}\right)\right\|_{L^{3}}+R e^{-1}\left\|\nabla\left(\mathbf{w}-\chi^{h}\right)\right\|^{2}+\beta\left\|\left(\mathbf{w}-\chi^{h}\right) \cdot \hat{\tau}_{j}\right\|_{\Gamma}^{2} .
\end{aligned}
$$

Using Young's inequality and the triangle inequality completes the proof.

Remark. $L_{p}$ stability estimates of the $L_{2}$ projection into finite element spaces is proven for many interesting spaces, for example, in [35] and [36].

\section{FINITE ELEMENT APPROXIMATION OF LARGE EDDY MOTION}

The usual, continuous-in-time, Galerkin finite element approximation of the solution of $(\mathbf{w}, q)$ of the large eddy model (1.1)-(1.4) will be given now. First, finite-dimensional finite element subspaces

$$
X^{h} \subset X, \quad Q^{h} \subset Q
$$

are selected that satisfy the discrete inf-sup condition (2.5).

The continuous-in-time approximations $\left(\mathbf{w}^{h}, q^{h}\right)$ are maps $\mathbf{w}^{h}:[0, T] \rightarrow X^{h}, q^{h}:(0, T] \rightarrow Q^{h}$ satisfying that $\mathbf{w}^{h}(0)$ approximates $\overline{\mathbf{u}}_{0}$ in $X^{h}$ and

$$
\begin{aligned}
\left(\mathbf{w}_{t}^{h}, \mathbf{v}^{h}\right)+\left(\left(R e^{-1}+\nu_{T}\left(\nabla \mathbf{w}^{h}\right)\right) \nabla \mathbf{w}^{h}, \nabla \mathbf{v}^{h}\right) & +b\left(\mathbf{w}^{h}, \mathbf{w}^{h}, \mathbf{v}^{h}\right)-\left(q^{h}, \nabla \cdot \mathbf{v}^{h}\right)+\beta(\delta, R e)\left(\mathbf{w}^{h} \cdot \hat{\tau}_{j}, \mathbf{v}^{h} \cdot \hat{\tau}_{j}\right)_{\Gamma} \\
& \left.-\mu_{1} \delta^{2}\left(\nabla \mathbf{w}^{h} \nabla \mathbf{w}^{h}\right), \nabla \mathbf{v}^{h}\right)=\left(\overline{\mathbf{f}}, \mathbf{v}^{h}\right)\left(\lambda^{h}, \nabla \cdot \mathbf{w}^{h}\right)=0,
\end{aligned}
$$

for all $\left(\mathbf{v}^{h}, \lambda^{h}\right) \in\left(X^{h}, Q^{h}\right)$. Using $V^{h}$ and the nonlinear operator $F(\cdot)$ defined by (2.3), we can write the approximation (3.1) more compactly. In particular, $\mathbf{w}^{h}:[0, T] \rightarrow V^{h}$ satisfies

$$
\begin{aligned}
\left(\mathbf{w}_{t}^{h}, \mathbf{v}^{h}\right)+\left(F\left(\mathbf{w}^{h}\right), \nabla\left(\mathbf{v}^{h}\right)\right)+b\left(\mathbf{w}^{h}, \mathbf{w}^{h},\right. & \left.\mathbf{v}^{h}\right) \\
& +\beta(\delta, \operatorname{Re})\left(\mathbf{w}^{h} \cdot \hat{\tau}_{j}, \mathbf{v}^{h} \cdot \hat{\tau}_{j}\right)_{\Gamma}=\left(\overline{\mathbf{f}}, \mathbf{v}^{h}\right), \text { for all } \mathbf{v}^{h} \in V^{h} .
\end{aligned}
$$

The method (3.1) or (3.2), respectively, is stable. It satisfies the same energy inequality as the continuous problem.

Proposition 3.1. [Leray's inequality for $\mathbf{w}^{h}$ ]. Suppose $\mathbf{w}^{h}$ is the solution of (3.2). Then, $\mathbf{w}^{h}$ satisfies for all $t>0$

$$
\begin{aligned}
\frac{1}{2}\left\|\mathbf{w}^{h}(t)\right\|^{2}+\int_{0}^{t}\left[\beta\left\|\mathbf{w}^{h} \cdot \hat{\tau}_{j}\right\|_{\Gamma}^{2}+R e^{-1}\left\|\nabla \mathbf{w}^{h}\right\|^{2}\right. & \\
& \left.+\underline{C} \mu_{0} \delta^{2}\left\|\nabla \mathbf{w}^{h}\right\|_{L^{3}}^{3}\right] d t^{\prime} \leq \frac{1}{2}\left\|\mathbf{w}^{h}(0)\right\|^{2}+\int_{0}^{t}\left(\overline{\mathbf{f}}\left(t^{\prime}\right), \mathbf{w}^{h}\left(t^{\prime}\right)\right) d t^{\prime} .
\end{aligned}
$$

In particular, 


$$
\int_{0}^{t} \operatorname{Re}^{-1}\left\|\nabla \mathbf{w}^{h}\right\|^{2} d t \leq\left\|\mathbf{w}^{h}(0)\right\|^{2}+C \operatorname{Re} \int_{0}^{t}\left\|\overline{\mathbf{f}}\left(t^{\prime}\right)\right\|^{2} d t^{\prime} .
$$

Proof. The proof of the first estimate is the same as that of Proposition 2.4. The second estimate follows from the first estimate by neglecting the first, second, and the last term on the left-hand side. Then, the second term on the right-hand side is estimated by the Cauchy-Schwarz inequality, the Poincaré inequality (2.2), and Young's inequality, leading to

$$
\int_{0}^{t}\left(\overline{\mathbf{f}}\left(t^{\prime}\right), \mathbf{w}^{h}\left(t^{\prime}\right)\right) d t^{\prime} \leq \int_{0}^{t}\left(\frac{\epsilon}{2}\left\|\overline{\mathbf{f}}\left(t^{\prime}\right)\right\|^{2}+\frac{C}{2 \epsilon}\left\|\mathbf{w}^{h}\left(t^{\prime}\right)\right\|^{2}\right) d t^{\prime} .
$$

Choosing $\epsilon=C R e$ and collecting terms, we conclude the proof.

By a similar argument, we obtain a particularly simple bound on $\left\|\mathbf{w}^{h}(t)\right\|$, uniform in both $R e$ and $\delta$.

Lemma 3.2. Suppose $\mathbf{w}^{h}$ is the solution of (3.2). Then, for $T>0$

$$
\max _{0 \leq t \leq T}\left\|\mathbf{w}^{h}(t)\right\| \leq\left\|\mathbf{w}^{h}(0)\right\|+\int_{0}^{T}\|\overline{\mathbf{f}}(t)\| d t
$$

Proof. Set $\mathbf{v}^{h}=\mathbf{w}^{h}$ in (3.2). Dropping the non-negative terms resulting on the left-hand side gives

$$
\frac{1}{2} \frac{d}{d t}\left\|\mathbf{w}^{h}(t)\right\|^{2}=\left\|\mathbf{w}^{h}\right\| \frac{d}{d t}\left\|\mathbf{w}^{h}(t)\right\| \leq\left(\overline{\mathbf{f}}, \mathbf{w}^{h}\right) \leq\|\overline{\mathbf{f}}\|\left\|\mathbf{w}^{h}\right\|
$$

Thus, $d / d t\left\|\mathbf{w}^{h}(t)\right\| \leq\|\overline{\mathbf{f}}(t)\|$, and the result follows.

Combining this lemma and Proposition 3.1 gives an a priori bound on the quantity

$$
a^{h}(t):=\left\|\mathbf{w}^{h}(t)\right\|^{1 / 2}\left\|\nabla \mathbf{w}^{h}(t)\right\|^{1 / 2} .
$$

Lemma 3.3. Let $a^{h}(t)$ be as above and $\mathbf{w}^{h}$ be the solution of (3.2). Then, $a^{h}(t) \in L^{4}(0, T)$ uniformly in $h$ and

$$
\left\|a^{h}(t)\right\|_{L^{4}(0, T)}^{4} \leq\left(\left\|\mathbf{w}^{h}(0)\right\|+\int_{0}^{T}\|\overline{\mathbf{f}}(t)\| d t\right)^{2}\left(\operatorname{Re}\left\|\mathbf{w}^{h}(0)\right\|^{2}+C \operatorname{Re}^{2} \int_{0}^{T}\|\bar{f}(t)\|^{2} d t\right) .
$$

Proof. Since

$$
\left\|a^{h}(t)\right\|_{L^{4}(0, T)}^{4} \leq\left\|\mathbf{w}^{h}\right\|_{L^{\infty}\left(0, T ; L^{2}(\Omega)\right)}^{2} \int_{0}^{T}\left\|\nabla \mathbf{w}^{h}\right\|^{2} d t
$$

the result follows from Proposition 3.1 and Lemma 3.2. 
The method (3.2) reduces existence of $\mathbf{w}^{h}$ to existence for a system of ordinary differential equations in $V^{h}$. The Cauchy-Schwarz inequality and Proposition 3.1 give immediately an a priori bound on $\mathbf{w}^{h}(t)$. Thus, $\mathbf{w}^{h}(x, t)$ exists uniquely. If the discrete inf-sup condition (2.5) and standard arguments of Girault and Raviart [30] are used, $q^{h}$ does as well.

We now turn to the error in the approximation $\mathbf{w}^{h}$ of $\mathbf{w}$. Important questions in the error analysis of large eddy simulation include dependence of the errors upon $R e$ and $\delta$, including cases in which $\delta$ and $h$ are related. In this report we consider only the first, without which later steps are not imaginable: we consider convergence of $\mathbf{w}^{h}$ to $\mathbf{w}$ as $h \rightarrow 0$ for $\operatorname{Re}$ and $\delta$ fixed. Further, if there were a convergence result for $\mathbf{w}^{h}$ to $\mathbf{w}$ that was uniformly in $\delta$, this would immediately imply a convergence result $\mathbf{w} \rightarrow \mathbf{u}$ (the solution of the underlying Navier-Stokes equations) as $\delta \rightarrow 0$. Such a result has never been proven (to the authors' knowledge) for any conventional turbulence model and only for the Camassa-Holm model and one large eddy model [37].

Theorem 3.4. Let $(\mathbf{w}, q)$ let be the solution of $(2.1)$ and $\left(\mathbf{w}^{h}, q^{h}\right)$ be the solution of (3.1). Let the finite element spaces fulfill the inf-sup condition (2.5), let $\mu_{0} \geq 4 \mu_{1}$, and let $a^{h}(t)$ be defined in (3.3). Under the assumption

$$
\nabla \mathbf{w} \in L^{4}\left(0, T ; L^{2}(\Omega)\right)
$$

the error $e=\mathbf{w}-\mathbf{w}^{h}$ satisfies

$$
\begin{aligned}
\|e\|_{L^{\infty}\left(0, T ; L^{2}\right)}^{2}+\beta\left\|e \cdot \hat{\tau}_{j}\right\|_{L^{2}\left(0, T ; L^{2}(\Gamma)\right)}^{2}+ & R e^{-1\|\nabla e\|_{L^{2}\left(0, T ; L^{2}\right)}^{2}+}+\mu_{0} \delta^{2}\|\nabla e\|_{L^{3}\left(0, T ; L^{3}\right)}^{3} \leq C C^{*}(T) \| \mathbf{w}(\mathbf{x}, 0) \\
& -\mathbf{w}^{h}(\mathbf{x}, 0) \|^{2}+C \inf _{\tilde{\mathbf{w}} \in X^{h}(\Omega), \lambda^{h} \in Q^{h}} \mathscr{F}\left(\mathbf{w}-\tilde{\mathbf{w}}, q-\lambda^{h}, R e, \delta, \mu_{0}, T\right)
\end{aligned}
$$

with

$$
C^{*}(T)=\exp \left(\int_{0}^{T}\left(1+C(R e)^{3}\|\nabla \mathbf{w}\|^{4}\right) d t\right)
$$

and

$$
\begin{aligned}
\mathscr{F}\left(\mathbf{w}-\tilde{\mathbf{w}}, q-\lambda^{h}, \operatorname{Re}, \delta, \mu_{0}, T\right)= & C\left[\|\mathbf{w}-\tilde{\mathbf{w}}\|_{L^{\infty}\left(0, T, L^{2}\right)}^{2}+\mu_{0} \delta^{2}\|\nabla(\mathbf{w}-\tilde{\mathbf{w}})\|_{L^{3}\left(0, T, L^{3}\right)}^{3}\right. \\
& +C^{*}(T)\left[\|(\mathbf{w}-\tilde{\mathbf{w}})(\mathbf{x}, 0)\|^{2}+\operatorname{Re}\left(\left\|a^{h}(t)\right\|_{L^{4}(0, T)}^{2}\right.\right. \\
& +\|\nabla \mathbf{w}\|_{L^{4}\left(0, T, L^{2}\right)}^{2}\|\nabla(\mathbf{w}-\tilde{\mathbf{w}})\|_{L^{4}\left(0, T, L^{2}\right)}^{2} \\
& +C\left(\delta, \mu_{0}, T\right)\|\nabla(\mathbf{w}-\tilde{\mathbf{w}})\|_{L^{3}\left(0, T, L^{3}\right)}^{3 / 2} \\
& +\operatorname{Re} e^{-1}\|\nabla(\mathbf{w}-\tilde{\mathbf{w}})\|_{L^{2}\left(0, T, L^{2}\right)}^{2}+\operatorname{Re}\left\|(\mathbf{w}-\tilde{\mathbf{w}})_{t}\right\|_{L^{2}\left(0, T, H^{-1}\right)}^{2} \\
& \left.\left.+\beta\left\|(\mathbf{w}-\tilde{\mathbf{w}}) \cdot \tau_{j}\right\|_{L^{2}\left(0, T, L^{2}(\Gamma)\right)}^{2}+\operatorname{Re}\left\|q-\lambda^{h}\right\|_{L^{2}\left(0, T, L^{2}\right)}^{2}\right]\right] .
\end{aligned}
$$

Proof. Let $e=\mathbf{w}-\mathbf{w}^{h}$ and $\mathbf{v}^{h} \in V^{h}$. An error equation is obtained by subtracting (3.1) from (2.1). This yields

$$
\begin{aligned}
\left(e_{t}, \mathbf{v}^{h}\right)+\left(F(\mathbf{w})-F\left(\mathbf{w}^{h}\right), \nabla \mathbf{v}^{h}\right)+b\left(\mathbf{w}, \mathbf{w}, \mathbf{v}^{h}\right) & \\
& -b\left(\mathbf{w}^{h}, \mathbf{w}^{h}, \mathbf{v}^{h}\right)+\beta\left(e \cdot \hat{\tau}_{j}, \mathbf{v}^{h} \cdot \hat{\tau}_{j}\right)_{\Gamma}=\left(q-\lambda^{h}, \nabla \cdot \mathbf{v}^{h}\right),
\end{aligned}
$$


where $\lambda^{h} \in Q^{h}$ is arbitrary. Let $\tilde{\mathbf{w}} \in V^{h}$ denote an approximation to $\mathbf{w}$. Then, with $\eta=\mathbf{w}-$ $\tilde{\mathbf{w}}$ and $\phi^{h}=\left(\mathbf{w}^{h}-\tilde{\mathbf{w}}\right) \in V^{h}$, and choosing $\mathbf{v}^{h}=\phi^{h}$, we can rewrite the error equation (3.5) as

$$
\begin{aligned}
\left(\phi_{t}^{h}, \phi^{h}\right)+\beta\left(\phi^{h} \cdot \hat{\tau}_{j}, \phi^{h} \cdot \hat{\tau}_{j}\right)_{\Gamma}+ & \left(F\left(\mathbf{w}^{h}\right)-F(\tilde{\mathbf{w}}), \nabla \phi^{h}\right)=b\left(\mathbf{w}, \mathbf{w}, \phi^{h}\right)-b\left(\mathbf{w}^{h}, \mathbf{w}^{h}, \phi^{h}\right)+(F(\mathbf{w}) \\
& \left.-F(\tilde{\mathbf{w}}), \nabla \phi^{h}\right)-\left(q-\lambda^{h}, \nabla \cdot \phi^{h}\right)+\left(\eta_{t}, \phi^{h}\right)+\beta\left(\eta \cdot \hat{\tau}_{j}, \phi^{h} \cdot \hat{\tau}_{j}\right)_{\Gamma} .
\end{aligned}
$$

Using Lemma 2.3 gives the estimate for the nonlinear eddy viscosity term

$\left(\left[\nu_{T}(\nabla \mathbf{w}) \nabla \mathbf{w}-\mu_{1} \delta^{2}(\nabla \mathbf{w} \nabla \mathbf{w})\right]-\left[\nu_{T}(\tilde{\mathbf{w}}) \nabla(\tilde{\mathbf{w}})\right.\right.$

$$
\left.\left.-\mu_{1} \delta^{2}(\nabla \tilde{\mathbf{w}} \nabla \tilde{\mathbf{w}})\right], \nabla \phi^{h}\right) \leq \bar{C} r \delta^{2}\|\nabla(\mathbf{w}-\tilde{\mathbf{w}})\|_{L^{3}}\left\|\nabla \phi^{h}\right\|_{L^{3}},
$$

with

$$
r=\max \left\{\|\nabla \mathbf{w}\|_{L^{3}},\|\nabla \tilde{\mathbf{w}}\|_{L^{3}}\right\} \leq C\|\nabla \mathbf{w}\|_{L^{3}}
$$

From Lemma 2.2 and the usual definition of a dual norm, we conclude

$$
\begin{aligned}
\frac{1}{2} \frac{d}{d t} \| & \phi^{h}\left\|^{2}+\beta\right\| \phi^{h} \cdot \hat{\tau}_{j}\left\|_{\Gamma}^{2}+R e^{-1}\right\| \nabla \phi^{h}\left\|^{2}+C \underline{\mu_{0}} \delta^{2}\right\| \nabla \phi^{h} \|_{L^{3}}^{3} \leq b\left(\mathbf{w}, \mathbf{w}, \phi^{h}\right)-b\left(\mathbf{w}^{h}, \mathbf{w}^{h}, \phi^{h}\right) \\
& +\left\|\eta_{t}\right\|_{H^{-1}}\left\|\nabla \phi^{h}\right\|+\bar{C} r \delta^{2}\|\nabla \eta\|_{L^{3}}\left\|\nabla \phi^{h}\right\|_{L^{3}}+R e^{-1}\|\nabla \eta\|\left\|\nabla \phi^{h}\right\|+\left\|q-\lambda^{h}\right\|\left\|\nabla \phi^{h}\right\| \\
& +\beta\left\|\eta \cdot \tau_{j}\right\|_{\Gamma}\left\|\phi^{h} \cdot \tau_{j}\right\|_{\Gamma} .
\end{aligned}
$$

We consider now the convection terms on the right-hand side of this last inequality. Adding and subtracting terms give

$$
b\left(\mathbf{w}, \mathbf{w}, \phi^{h}\right)-b\left(\mathbf{w}^{h}, \mathbf{w}^{h}, \phi^{h}\right)=b\left(\mathbf{w}, e, \phi^{h}\right)+b\left(e, \mathbf{w}, \phi^{h}\right)-b\left(e, e, \phi^{h}\right) .
$$

By skew symmetry and $e=\eta-\phi^{h}=\mathbf{w}-\mathbf{w}^{h}$, this can be rewritten in the form

$$
b\left(\mathbf{w}, \mathbf{w}, \phi^{h}\right)-b\left(\mathbf{w}^{h}, \mathbf{w}^{h}, \phi^{h}\right)=b\left(\eta, \mathbf{w}, \phi^{h}\right)+b\left(\mathbf{w}^{h}, \eta, \phi^{h}\right)-b\left(\phi^{h}, \mathbf{w}, \phi^{h}\right) .
$$

In the analysis of the trilinear form we will use the estimate

$$
b(\mathbf{u}, \mathbf{v}, \mathbf{w}) \leq C\|\nabla \mathbf{u}\|^{1 / 2}\|\mathbf{u}\|^{1 / 2}\|\nabla \mathbf{v}\|\|\nabla \mathbf{w}\|
$$

This estimate is derived by Hölder's inequality, the Sobolev imbeddings $W^{1 / 2,2}(\Omega) \rightarrow L^{3}(\Omega)$ and $W^{1,2}(\Omega) \rightarrow L^{6}(\Omega)$, the interpolation of $W^{1 / 2,2}(\Omega)$ by $L^{2}(\Omega)$ and $W^{1,2}(\Omega)$, and Poincaré's inequality (2.2); see also the proof of Lemma 2.2 (f) in [38]. We obtain by (3.9), Poincaré's inequality (2.2), and Young's inequality, for any $\epsilon>0$

$$
\begin{aligned}
\left|b\left(\eta, \mathbf{w}, \phi^{h}\right)+b\left(\mathbf{w}^{h}, \eta, \phi^{h}\right)\right| & \leq C\left(\|\nabla \eta\|^{1 / 2}\|\eta\|^{1 / 2}\|\nabla \mathbf{w}\|+\left\|\nabla \mathbf{w}^{h}\right\|^{1 / 2}\left\|\mathbf{w}^{h}\right\|^{1 / 2}\|\nabla \eta\|\right)\left\|\nabla \phi^{h}\right\| \\
& \leq C\left(\|\nabla \mathbf{w}\|+\left\|\nabla \mathbf{w}^{h}\right\|^{1 / 2}\left\|\mathbf{w}^{h}\right\|^{1 / 2}\right)\|\nabla \eta\|\left\|\nabla \phi^{h}\right\| \\
& \leq \frac{\epsilon}{2}\left\|\nabla \phi^{h}\right\|^{2}+\frac{C}{\epsilon}\left(\|\nabla \mathbf{w}\|^{2}+\left\|\nabla \mathbf{w}^{h}\right\|\left\|\mathbf{w}^{h}\right\|\right)\|\nabla \eta\|^{2} .
\end{aligned}
$$


The remaining term on the right-hand side of (3.8) is also estimated by (3.9) and Young's inequality:

$$
\left|b\left(\phi^{h}, \mathbf{w}, \phi^{h}\right)\right| \leq C\left\|\nabla \phi^{h}\right\|^{3 / 2}\left\|\phi^{h}\right\|^{1 / 2}\|\nabla \mathbf{w}\| \leq \frac{\epsilon}{2}\left\|\nabla \phi^{h}\right\|^{2}+\frac{C}{\epsilon^{3}}\|\nabla \mathbf{w}\|^{4}\left\|\phi^{h}\right\|^{2} .
$$

Applying again Young's inequality, for $\epsilon=\underline{C} \mu_{0}$,

$$
\begin{aligned}
\bar{C} r \delta^{2}\left\|\nabla \phi^{h}\right\|_{L^{3}}\|\nabla \eta\|_{L^{3}} & \leq \frac{\epsilon}{3} \delta^{2}\left\|\nabla \phi^{h}\right\|_{L^{3}}^{3}+\frac{2}{3} \epsilon^{-1 / 2} \delta^{2}(\bar{C} r)^{3 / 2}\|\nabla \eta\|_{L^{3}}^{3 / 2} \\
& =\frac{1}{3} \underline{C} \mu_{0} \delta^{2}\left\|\nabla \phi^{h}\right\|_{L^{3}}^{3}+\frac{2}{3}(\bar{C} r)^{3 / 2}\left(\underline{C} \mu_{0}\right)^{-1 / 2} \delta^{2}\|\nabla \eta\|_{L^{3}}^{3 / 2} .
\end{aligned}
$$

The remaining terms on the right-hand side of (3.7) are also estimated by Young's inequality:

$$
\begin{gathered}
\left\|\eta_{t}\right\|_{H^{-1}}\left\|\nabla \phi^{h}\right\| \leq \operatorname{Re}\left\|\eta_{t}\right\|_{H^{-1}}^{2}+\frac{R e^{-1}}{4}\left\|\nabla \phi^{h}\right\|^{2} \\
R e^{-1}\|\nabla \eta\|\left\|\nabla \phi^{h}\right\| \leq \frac{1}{2 \operatorname{Re}}\|\nabla \eta\|^{2}+\frac{1}{2 \operatorname{Re}}\left\|\nabla \phi^{h}\right\|^{2} \\
\left\|q-\lambda^{h}\right\|\left\|\nabla \phi^{h}\right\| \leq \frac{1}{2 \epsilon}\left\|q-\lambda^{h}\right\|^{2}+\frac{\epsilon}{2}\left\|\nabla \phi^{h}\right\|^{2} \\
\beta\left\|\eta \cdot \tau_{j}\right\|_{\Gamma}\left\|\phi^{h} \cdot \tau_{j}\right\|_{\Gamma} \leq \frac{\beta}{2}\left\|\eta \cdot \tau_{j}\right\|_{\Gamma}^{2}+\frac{\beta}{2}\left\|\phi^{h} \cdot \tau_{j}\right\|_{\Gamma}^{2}
\end{gathered}
$$

Picking $\epsilon=O\left(R e^{-1}\right)$, inserting all estimates into (3.7), and collecting terms give

$$
\begin{aligned}
\frac{1}{2} \frac{d}{d t} \| & \phi^{h}\left\|^{2}+\frac{\beta}{2}\right\| \phi^{h} \cdot \hat{\tau}_{j}\left\|_{\Gamma}^{2}+C R e^{-1}\right\| \nabla \phi^{h}\left\|^{2}+\frac{2}{3} \underline{C} \mu_{0} \delta^{2}\right\| \nabla \phi^{h} \|_{L^{3}}^{3} \leq C\left[\operatorname { R e } \left(\|\nabla \mathbf{w}\|^{2}\right.\right. \\
+ & \left.\left\|\nabla \mathbf{w}^{h}\right\|\left\|\mathbf{w}^{h}\right\|\right)\|\nabla \eta\|^{2}+\operatorname{Re}\left\|\eta_{t}\right\|^{2}+\frac{1}{2 \operatorname{Re}}\|\nabla \eta\|^{2}+\frac{2}{3}(\bar{C} r)^{3 / 2}\left(\underline{C} \mu_{0}\right)^{-1 / 2} \delta^{2}\|\nabla \eta\|_{L^{3}}^{3 / 2}+C \operatorname{Re} \| q \\
& \left.-\lambda^{h}\left\|^{2}+\frac{\beta}{2}\right\| \eta \cdot \tau_{j} \|_{\Gamma}^{2}\right]+\left[\frac{1}{2}+C(R e)^{3}\|\nabla \mathbf{w}\|^{4}\right]\left\|\phi^{h}\right\|^{2} .
\end{aligned}
$$

Since, by assumption, $\|\nabla \mathbf{w}\|^{4} \in L^{1}(0, T)$, Gronwall's inequality now implies

$$
\begin{aligned}
\max _{0 \leq t \leq T} & \left\|\phi^{h}\right\|^{2}+\int_{0}^{T}\left(2 \beta\left\|\phi^{h} \cdot \hat{\tau}_{j}\right\|_{\Gamma}^{2}+R e^{-1}\left\|\nabla \phi^{h}\right\|^{2}+\frac{4}{3} \underline{C} \mu_{0} \delta^{2}\left\|\nabla \phi^{h}\right\|_{L^{3}}^{3}\right) d t \leq C^{*}(T)\left\|\phi^{h}(0)\right\|^{2} \\
& +C C^{*}(T) \int_{0}^{T}\left(\operatorname{Re}\left(\|\nabla \mathbf{w}\|^{2}+\left\|\nabla \mathbf{w}^{h}\right\|\left\|\mathbf{w}^{h}\right\|\right)\|\nabla \eta\|^{2}+R e\left\|\eta_{t}\right\|^{2}+\frac{1}{\operatorname{Re}}\|\nabla \eta\|^{2}+r^{3 / 2} \mu_{0}^{-1 / 2} \delta^{2}\|\nabla \eta\|_{L^{3}}^{3 / 2}\right. \\
& \left.+\operatorname{Re}\left\|q-\lambda^{h}\right\|^{2}+\beta\left\|\eta \cdot \tau_{j}\right\|_{\Gamma}^{2}\right) d t
\end{aligned}
$$


TABLE I. Example 1, mesh widths and degrees of freedom for $Q_{2} / P_{1}^{\text {disc }}$ discretization (left) and $Q_{3} / P_{2}^{\text {disc }}$ discretization (right).

\begin{tabular}{lrrrrrr}
\hline$h$ & Velocity & Pressure & Total & Velocity & Pressure & Total \\
\hline $1 / 4$ & & & & 338 & 96 & 434 \\
$1 / 8$ & 578 & 192 & 770 & 1250 & 384 & 1634 \\
$1 / 16$ & 2178 & 768 & 2946 & 4802 & 1536 & 6338 \\
$1 / 32$ & 8450 & 3072 & 11522 & 18818 & 6144 & 24962 \\
$1 / 64$ & 33282 & 12288 & 45570 & & & \\
\hline
\end{tabular}

where $C^{*}(T)$ is defined in (3.4). We can bound by using the Cauchy-Schwarz inequality in $L^{2}(0$, T):

$$
\begin{aligned}
\int_{0}^{T}\|\nabla \mathbf{w}\|^{2}\|\nabla \eta\|^{2} d t & \leq\|\nabla \mathbf{w}\|_{L^{4}\left(0, T, L^{2}\right)}^{2}\|\nabla \eta\|_{L^{4}\left(0, T, L^{2}\right)}^{2} \\
\int_{0}^{T}\left\|\nabla \mathbf{w}^{h}\right\|\left\|\mathbf{w}^{h}\right\|\|\nabla \eta\|^{2} & \leq\left\|a^{h}(t)\right\|_{L^{4}(0, T)}\|\nabla \eta\|_{L^{4}\left(0, T, L^{2}\right)}^{2} \\
\int_{0}^{T} r^{3 / 2}\|\nabla \eta\|_{L^{3}}^{3 / 2} & \leq C \int_{0}^{T}\|\nabla \mathbf{w}\|_{L^{3}}^{3 / 2}\|\nabla \eta\|_{L^{3}}^{3 / 2} \leq C\|\nabla \mathbf{w}\|_{L^{3}\left(0, T, L^{3}\right)}^{3 / 2}\|\nabla \eta\|_{L^{3}\left(0, T, L^{3}\right)}^{3 / 2} \\
& \leq C\left(\delta, \mu_{0}, T\right)\|\nabla \eta\|_{L^{3}\left(0, T, L^{3}\right)}^{3 / 2} .
\end{aligned}
$$

The last estimate was obtained by using the estimate of $r$ (3.6) and the result of Proposition 2.4. The term $a^{h}(t)$ is estimated in Lemma 3.3.

The error estimate now follows by the triangle inequality and collecting terms.

\section{NUMERICAL RESULTS}

We present two numerical tests that confirm the error estimate given in Theorem 3.4. The first example is Chorin's vortex decay problem in $2 \mathrm{D}$, and the second example has an analytical solution in 3D. A third example illustrates the slip with resistance boundary condition. Before the numerical examples, we will describe the numerical schemes used in the computations.

\begin{tabular}{|c|c|c|c|c|c|c|}
\hline \multirow[b]{2}{*}{$h$} & \multicolumn{2}{|l|}{$\tau=1000$} & \multirow{2}{*}{$\begin{array}{c}\tau=10,000 \\
\text { Error }\end{array}$} & \multicolumn{3}{|c|}{$\tau=100,000$} \\
\hline & Error & Order & & Order & Error & Order \\
\hline $1 / 8$ & $2.139817 \mathrm{e}-02$ & & $2.140299 \mathrm{e}-02$ & & $2.140347 \mathrm{e}-02$ & \\
\hline $1 / 16$ & $2.758204 \mathrm{e}-03$ & 2.956 & $2.758924 \mathrm{e}-03$ & 2.956 & $2.758996 \mathrm{e}-03$ & 2.956 \\
\hline $1 / 32$ & $3.470081 \mathrm{e}-04$ & 2.991 & $3.470864 \mathrm{e}-04$ & 2.991 & $3.471288 \mathrm{e}-04$ & 2.991 \\
\hline $1 / 64$ & $4.498844 \mathrm{e}-05$ & 2.947 & $4.351145 \mathrm{e}-05$ & 2.996 & $4.351082 \mathrm{e}-05$ & 2.996 \\
\hline
\end{tabular}

We start by discretizing Equation (1.1) in time by the fractional-step- $\theta$-scheme; see, for example, Bristeau, Glowinsky, and Periaux [39], which is analyzed for the time-dependent

TABLE II. Example $1,\|e\|_{L^{\infty}\left(0, T, L^{2}\right)}$ for the $Q_{2} / P_{1}^{\text {disc }}$ discretization. 
TABLE III. Example 1, $\|\nabla e\|_{L^{2}\left(0, T, L^{2}\right)}$ for the $Q_{2} / P_{1}^{\text {disc }}$ discretization.

\begin{tabular}{|c|c|c|c|c|c|c|}
\hline \multicolumn{3}{|c|}{$\tau=1000$} & \multicolumn{2}{|l|}{$\tau=10,000$} & \multicolumn{2}{|l|}{$\tau=100,000$} \\
\hline$h$ & Error & Order & Error & Order & Error & Order \\
\hline $1 / 8$ & $1.476035 \mathrm{e}+00$ & & $3.954061 \mathrm{e}+00$ & & $5.086226 \mathrm{e}+00$ & \\
\hline $1 / 16$ & $3.652900 \mathrm{e}-01$ & 2.015 & $9.784881 \mathrm{e}-01$ & 2.015 & $1.258638 \mathrm{e}+00$ & 2.015 \\
\hline $1 / 32$ & $9.097928 \mathrm{e}-02$ & 2.005 & $2.436802 \mathrm{e}-01$ & 2.006 & $3.134455 \mathrm{e}-01$ & 2.006 \\
\hline $1 / 64$ & $2.273662 \mathrm{e}-02$ & 2.001 & $6.087638 \mathrm{e}-02$ & 2.001 & $7.830957 \mathrm{e}-02$ & 2.001 \\
\hline
\end{tabular}

Navier-Stokes equations by Kloucek and Rys [40]. This implicit scheme is of second-order accuracy, more stable than the Crank-Nicolson scheme, and is currently considered "best" on the basis of accuracy and reliability; see, for example, Turek [41] or Rannacher [42].

The fractional-step- $\theta$-scheme divides each time step into three subtime steps. In each subtime step, a nonlinear saddle point problem has to be solved. The nonlinear problem is linearized by a fixed-point iteration. The term coming from the LES model, $A^{-1}(\nabla \mathbf{w} \nabla \mathbf{w})$, is computed only at the beginning of each subtime step and is not changed during the fixed-point iteration. All other nonlinear terms are updated after each iteration step. We have used two finite element discretizations of different polynomial degree to discretize the arising linear saddle point problems. The lower-order finite element is the $Q_{2} / P_{1}^{\text {disc }}$ (or $Q_{2} / P_{-1}$ in the notation of Gresho and Sani [11]) finite element discretization; that is, the velocity is approximated by continuous piecewise biquadratics in 2D (triquadratics in 3D) and the pressure by discontinuous linears. The higher-order pair of finite elements is the $Q_{3} / P_{2}^{\text {disc }}$ discretization; that is the discrete velocity is continuous and piecewise bicubic in 2D (tricubic in 3D) and the pressure discontinuous and piecewise quadratic. These conforming pairs of finite element spaces fulfill the inf-sup or Babuška-Brezzi stability condition on meshes consisting of parallelepipeds. They are currently considered among the most stable and best-performing elements for finite element discretizations of Navier-Stokes equations; see, for example, Fortin [43], Gresho and Sani [11], or the studies in $[44,45]$.

The linear saddle point problems are solved by a flexible GMRES method; see Saad [46]. The preconditioner is a coupled multigrid method with Vanka-type smoothers as studied numerically, for example, in [47, 44, 45]. These algorithmic choices are currently considered among the best in terms of reliability, stability, and accuracy in finite element CFD.

Example 1. Chorin's vortex decay problem in 2D.This problem is taken from [48] and is also used in other numerical tests, for example, by Tafti [49] or in [22]. The domain is the unit square $\Omega=(0,1)^{2}$, and the prescribed solution has the form

$$
\begin{aligned}
& w_{1}=-\cos (n \pi x) \sin (n \pi y) \exp \left(-2 n^{2} \pi^{2} t / \tau\right), \\
& w_{2}=\sin (n \pi x) \cos (n \pi y) \exp \left(-2 n^{2} \pi^{2} t / \tau\right),
\end{aligned}
$$

\begin{tabular}{|c|c|c|c|c|c|c|}
\hline \multirow[b]{2}{*}{$h$} & \multicolumn{2}{|l|}{$\tau=1000$} & \multirow{2}{*}{$\tau=10,000$} & \multicolumn{3}{|c|}{$\tau=100,000$} \\
\hline & Error & Order & & Order & Error & Order \\
\hline $1 / 4$ & $3.301491 \mathrm{e}-02$ & & $3.497423 e-02$ & & $3.531723 \mathrm{e}-02$ & \\
\hline $1 / 8$ & $2.065646 \mathrm{e}-03$ & 3.998 & $2.066067 \mathrm{e}-03$ & 4.081 & $2.066109 \mathrm{e}-03$ & 4.095 \\
\hline $1 / 16$ & $1.364503 e-04$ & 3.920 & $1.364647 \mathrm{e}-04$ & 3.920 & $1.364567 \mathrm{e}-04$ & 3.920 \\
\hline $1 / 32$ & $8.900156 \mathrm{e}-06$ & 3.938 & $8.902196 \mathrm{e}-06$ & 3.938 & $8.902265 \mathrm{e}-06$ & 3.938 \\
\hline
\end{tabular}

TABLE IV. Example 1, $\|e\|_{L^{\infty}\left(0, T, L^{2}\right)}$ for the $Q_{3} / P_{2}^{\text {disc }}$ discretization. 
TABLE V. Example $1,\|\nabla e\|_{L^{2}\left(0, T, L^{2}\right)}$ for the $Q_{3} / P_{2}^{\text {disc }}$ discretization.

\begin{tabular}{|c|c|c|c|c|c|c|}
\hline \multirow[b]{2}{*}{$h$} & \multicolumn{2}{|l|}{$\tau=1000$} & \multirow{2}{*}{$\begin{array}{c}\tau=10,000 \\
\text { Error }\end{array}$} & \multicolumn{3}{|c|}{$\tau=100,000$} \\
\hline & Error & Order & & Order & Error & Order \\
\hline $1 / 4$ & $1.555536 \mathrm{e}+00$ & & $4.164188 \mathrm{e}+00$ & & $5.356372 \mathrm{e}+00$ & \\
\hline $1 / 8$ & $2.156786 \mathrm{e}-01$ & 2.850 & $5.778960 \mathrm{e}-01$ & 2.849 & 7.434777e-01 & 2.849 \\
\hline $1 / 16$ & $2.549815 \mathrm{e}-02$ & 3.080 & $6.814509 \mathrm{e}-02$ & 3.084 & $8.765900 \mathrm{e}-02$ & 3.084 \\
\hline $1 / 32$ & $3.160856 \mathrm{e}-03$ & 3.012 & $8.432707 \mathrm{e}-03$ & 3.015 & $1.084991 \mathrm{e}-02$ & 3.014 \\
\hline
\end{tabular}

$$
q=-\frac{1}{4}(\cos (2 n \pi x)+\cos (2 n \pi y)) \exp \left(-4 n^{2} \pi^{2} t / \tau\right)
$$

We have chosen $n=4$ and three different values of the relaxation parameter $\tau$ in our computations. Because the solution is known, we use for simplicity Dirichlet boundary conditions. The right-hand side $\overline{\mathbf{f}}$ is chosen such that $\mathbf{w}=\left(w_{1}, w_{2}\right)^{T}$ and $q$ fulfill

$$
\mathbf{w}_{t}-R e^{-1} \Delta \mathbf{w}+\mathbf{w} \cdot \nabla \mathbf{w}+\nabla q-\nabla \cdot\left(\mu_{0} \delta^{2}|\nabla \mathbf{w}| \nabla \mathbf{w}\right)+\mu_{1} \delta^{2} \nabla \cdot(\nabla \mathbf{w} \nabla \mathbf{w})=\overline{\mathbf{f}} \text { in }(0, T] \times \Omega .
$$

The derivation of the Taylor LES model with the Gaussian filter gives $\mu_{1}=1 / 12$; see, for example, [14]. As consequence of Lemma 2.2, we have chosen $\mu_{0}=1 / 3$. The other parameters in this example were

$$
R e=10000, \quad \delta=0.1, \quad T=20
$$

To reduce the influence of the time discretization error, we carried out all computations with the small equidistant time step $\Delta t=0.001$. The unit square was decomposed into equidistant grids with squares of size $h \times h$. The number of degrees of freedom (d.o.f.) for the finite element discretizations and the different mesh sizes are given in Table I.

We present results for $\|e\|_{L^{\infty}\left(0, T, L^{2}\right)}$, Tables II and IV, and for $\|\nabla e\|_{L^{2}\left(0, T, L^{2}\right)}$, Tables III and V. According to Theorem 3.4, the order of convergence of these errors is connected to the approximation error of the used pair of finite elements. This can be clearly observed in the numerical results. Theorem 3.4 predicts the same order of convergence for $\|e\|_{L^{\infty}\left(0, T, L^{2}\right)}$ and $\|\nabla e\|_{L^{2}\left(0, T, L^{2}\right)}$. The higher order of convergence for $\|e\|_{L^{\infty}\left(0, T, L^{2}\right)}$ in the numerical results can be explained with the smoothness of the prescribed solution, because the error estimate for $\|e\|_{L^{\infty}\left(0, T, L^{2}\right)}$ can be improved assuming a higher regularity of $\mathbf{w}$ in Theorem 3.4.

In addition, under the assumption that the approximation errors in $\mathscr{F}\left(\mathbf{w}-\tilde{\mathbf{w}}, q-\lambda^{h}, R e\right.$, $\left.\delta, \mu_{0}, T\right)$ are independent of $t$, Theorem 3.4 predicts a rate of convergence of $O\left(h^{3 k / 4}\right)$, where $k$ is the polynomial degree of the finite element velocity space. This order comes from

TABLE VI. Example 2, mesh widths and degrees of freedom for $Q_{2} / P_{1}^{\text {disc }}$ discretization.

\begin{tabular}{lrrr}
\hline$h$ & Velocity & Pressure & Total \\
\hline $1 / 2$ & 375 & 32 & 407 \\
$1 / 4$ & 2187 & 256 & 2443 \\
$1 / 8$ & 14739 & 2048 & 16787 \\
$1 / 16$ & 107811 & 16384 & 124195 \\
\hline
\end{tabular}


TABLE VII. Example 2, $\|e\|_{L^{\infty}\left(0, T, L^{2}\right)}$ (left) and $\|\nabla e\|_{L^{2}\left(0, T, L^{2}\right)}$ (right).

\begin{tabular}{llccr}
\hline & $\|e\|_{L^{\infty}\left(O, T, L^{2}\right)}$ & Order & $\|\nabla e\|_{L^{2}\left(0, T, L^{2}\right)}$ & Order \\
\hline & Error & & Error & \\
$1 / 2$ & $3.080651 \mathrm{e}+00$ & 3.520 & $1.055479 \mathrm{e}+02$ & 2.671 \\
$1 / 4$ & $2.686117 \mathrm{e}-01$ & 2.700 & $1.657113 \mathrm{e}+01$ & 1.835 \\
$1 / 8$ & $4.133357 \mathrm{e}-02$ & 2.685 & $1.645197 \mathrm{e}+00$ & 2.143 \\
$1 / 16$ & $6.429026 \mathrm{e}-03$ & & $1.051972 \mathrm{e}+00$ & \\
\hline
\end{tabular}

the term $\left.\|\nabla(\mathbf{w}-\tilde{\mathbf{w}})\|_{L^{3}}^{3 / 2} 0, T, L^{3}\right)$. The computations on this very smooth problem show $O\left(h^{k}\right)$ and even $O\left(h^{k+1}\right)$ convergence. The asymptotic error estimate $O\left(h^{3 k / 4}\right)$ in Theorem 3.4 includes more general nonsmooth problems. This reduced rate of convergence appears already in Proposition 2.5 and even in usual energy norm error estimates for the $\mu$-Laplacian alone.

Example 2. An analytical solution in 3D.In this example, the right-hand side $\overline{\mathbf{f}}$ is chosen such that

$$
\begin{aligned}
& w_{1}=t(10-t)\left(x^{2}+y^{2}+z^{2}+y^{5}+\sin (\pi x) \sin (\pi y) \sin (\pi z)\right), \\
& w_{2}=t(10-t)\left(x^{2}+2 x z+3 z^{4}+13+\cos (\pi x) \cos (\pi y) \cos (\pi z)\right), \\
& w_{3}=t(10-t)\left(-2 x z+5 y^{2}-x^{4} y+\cos (\pi x) \sin (\pi y) \cos (\pi z)+\cos (\pi x) \sin (\pi y) \sin (\pi z)\right), \\
& q=t(10-t)(3 x-2 y+7 z-4) / 25,
\end{aligned}
$$

fulfill (4.1) in $\Omega=(0,1)^{3}$. Again, we used for simplicity Dirichlet boundary conditions. The other parameters in this example were

$$
\mu_{0}=\frac{1}{3}, \quad \mu_{1}=\frac{1}{12}, R e=10,000, \quad \delta=0.1, \quad T=10 .
$$

The solution depends quadratically on $t$ such that the discretization error in time of the fractional-step- $\theta$ scheme is negligible. We have used the equidistant time step $\Delta t=0.01$. The unit cube is divided into equidistant $h \times h \times h$ meshes. The number of degrees of freedom for the different mesh sizes using the $Q_{2} / P_{1}^{\text {disc }}$ discretization is given in Table VI.

The results for $\|e\|_{L^{\infty}\left(0, T, L^{2}\right)}$ and $\|\nabla e\|_{L^{2}\left(0, T, L^{2}\right)}$ are presented in Table VII. The evaluation of the numerical tests leads to the same observations as in Example 1.

Example 3. We like to give with this example a numerical illustration of the application of the slip with resistance and no penetration boundary conditions. To our knowledge, these

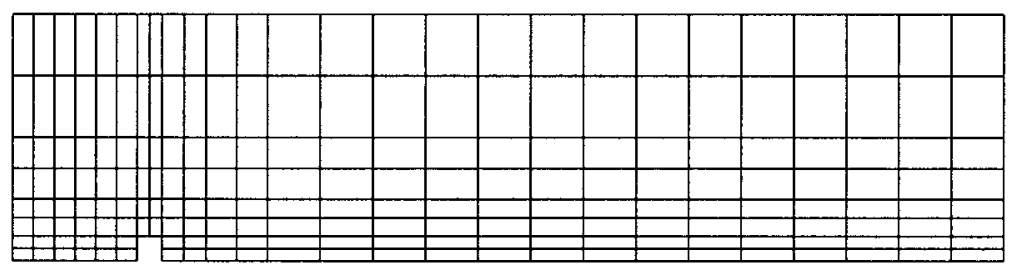

FIG. 1. Coarsest grid (level 0) in Example 3. 

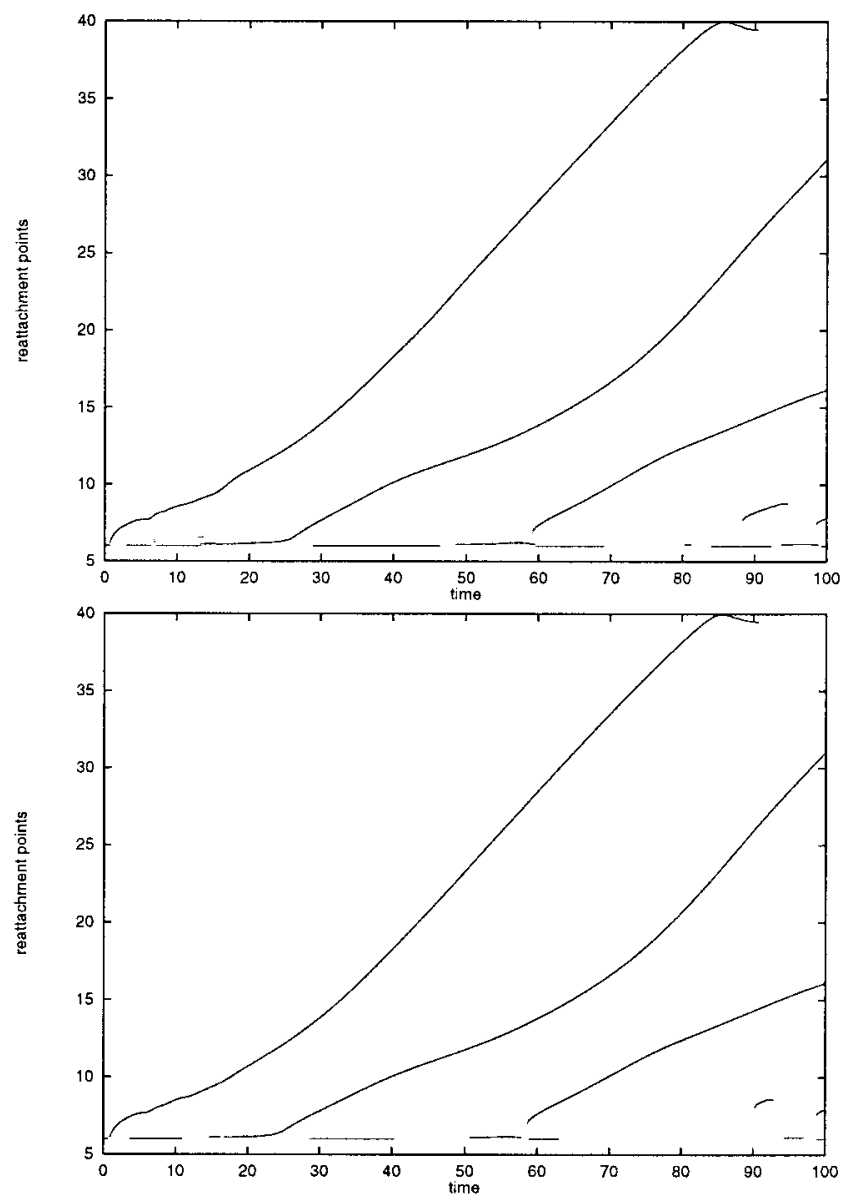

FIG. 2. Example 3, reattachment points for slip with friction boundary conditions (top) and no-slip boundary conditions (bottom).

boundary conditions have not been used in LES before. The implementation of these boundary conditions into a finite element code is described in [50]. In addition, we wish to demonstrate the behavior of the regularization operator $A^{-1}$ in (1.1). An assessment of some models in LES, [51], demonstrates that the regularization the Taylor LES model, $A^{-1}=I$, and leads in numerical tests often to a blowup of the solution. In contrast, $A^{-1}=\left(I-\delta^{2} /(4 \gamma) \Delta\right)^{-1}$ and $A^{-1}=g_{\delta} *$, both called the rational LES model in [51], have yielded good numerical results.

We consider the channel flow past a step; see Fig. 1 for the domain and the initial grid. The length of the channel is 40, its height 10; the step of height 1 starts at length 5 and ends at length 6. Parabolic inflow boundary conditions

$$
\mathbf{w}=(y(10-y) / 25,0)^{T}
$$

and outflow boundary conditions, so-called do-nothing conditions, (see Heywood, Rannacher, and Turek [52]), are prescribed. The parameters in (1.1) and (1.2) are chosen to be 

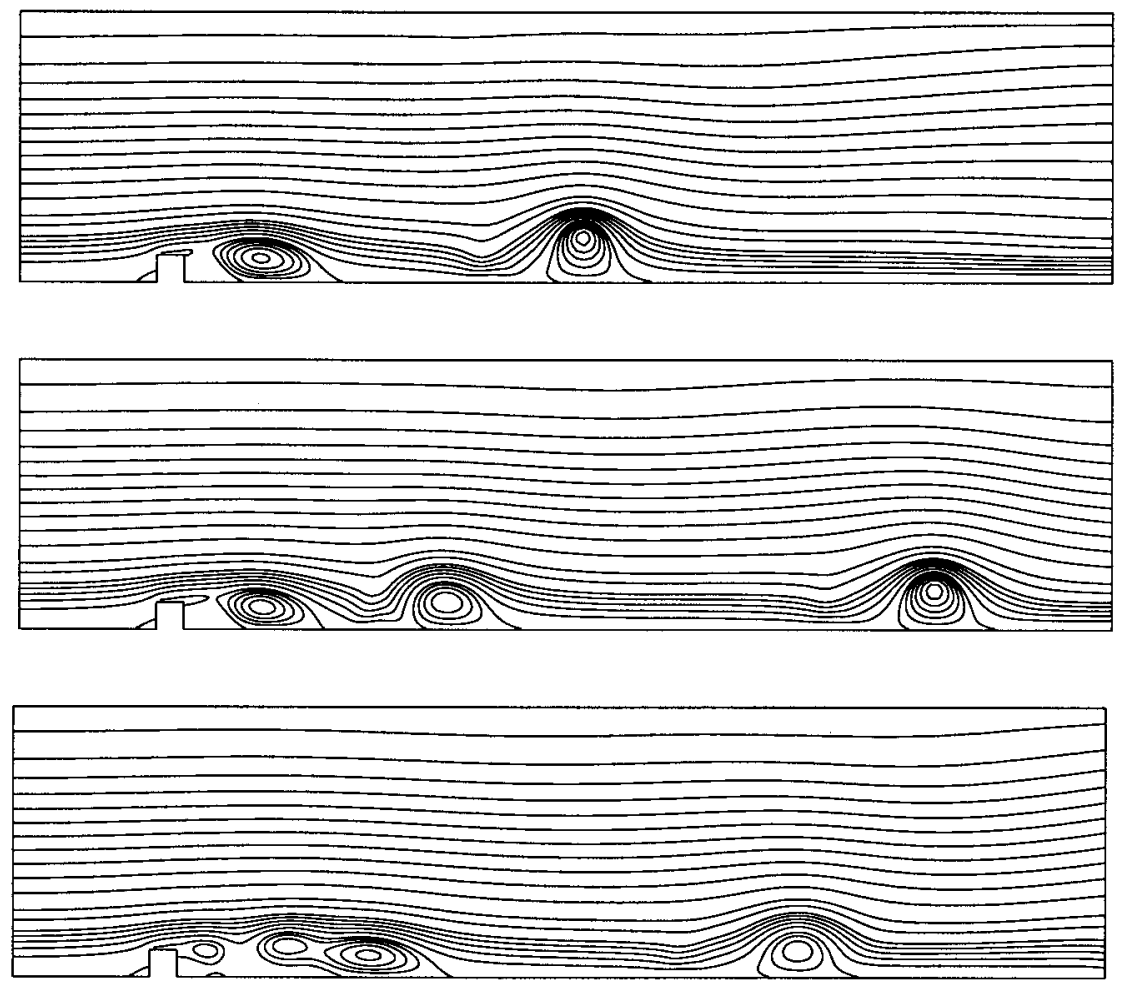

FIG. 3. Example 3, streamlines for $t=50,75,100$.

$$
\begin{aligned}
R e & =1000, \quad \delta=0.0625, \quad \mu_{0}=0.01, \quad \mu_{1} \\
& =\frac{1}{12}, \quad T=100, \quad A^{-1}=\left(I-\frac{\delta^{2}}{4 \gamma} \Delta\right)^{-1} .
\end{aligned}
$$

The friction constant in (1.4) was set $\beta=0.1$.

We test the qualitative fidelity of the finite element approximation using $\mu_{0}=0.01, \mu_{1}=$ $1 / 12$. These values lie outside the regime $\mu_{0} \geq 4 \mu_{1}$ for which full mathematical reliability can be proven for the Taylor LES model. Although the numerical analysis of the model with regularization, the rational LES model, is not yet complete, the available theory, [3], and computational experience, [51], suggest the extra $A^{-1}$-regularization will ensure a reliable simulation. Indeed, with regularization, the finite element approximation works smoothly in this parameter regime.

We used the $Q_{2} / P_{1}^{\text {disc }}$ finite element discretization in this example. The computations were carried out on level 3 (105 602 d.o.f. of the velocity, 39168 d.o.f. of the pressure) and with equal distant time step $\Delta t=0.01$.

The initial velocity was set to be zero in all interior degrees of freedom and we applied an impulsive start. The flow starts to develop vortices behind the step which are traveling on the lower boundary and finally leaving the channel. Because of the slip with resistance boundary condition, the reattachment point of a vortex can be determined easily by a change of the sign of the tangential velocity on the boundary. In contrast to homogeneous Dirichlet boundary 


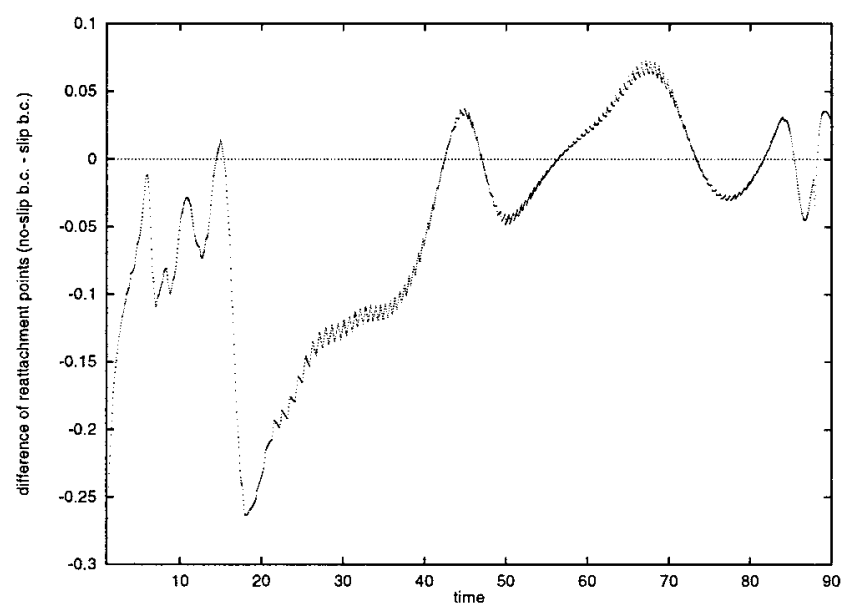

FIG. 4. Example 3, difference in the reattachment points (no-slip boundary condition - slip boundary condition) for the first traveling eddy.

conditions, the tangential component, in general, does not vanish. The reattachment points of the vortices are presented in Fig. 2 for $t \in[0,100]$. Streamlines of the corresponding velocity for some times $t$ are given in Figure 3 .

The reattachment points for a computation with no-slip boundary conditions are also presented in Fig. 2. On the scale of Fig. 2, one can observe only minor differences to the solution with the slip with friction boundary condition. However, a closer look on the difference of the reattachment points of the first travelling eddy, Fig. 4, shows clearly the different dynamics in the evolution of these points. A detailed investigation of the effect of different friction parameters on the flow is beyond the scope of this article and will be subject of a forthcoming study.

\section{References}

1. P. Galdi and W. Layton, Approximation of the larger eddies in fluid motion ii: A model for space filtered flow, Math Models Meth Appl Sci 10(3) (2000), 343-350.

2. T. Iliescu and W. J. Layton, Approximating the larger eddies in fluid motion iii: the Boussinesq model for turbulent fluctuations, An St Univ "Al I Cuza" Iaşi, 44 (1998), 245-261.

3. R. Lewandowski, Analyse Mathematique et Oceanographie, Masson, 1997.

4. J. S. Smagorinsky, General circulation experiments with the primitive equations, Mon Weather Rev 91 (1963), 99-164.

5. W. J. Layton, A nonlinear, subgridscale model for incompressible viscous flow problems, SIAM J Sci Comput 17(2) (1996), 347-357.

6. W. J. Layton, Subgridscale modelling and finite element methods for the Navier Stokes equations, Report mbi-96-4, Otto-von-Guericke Universität, Magdeburg, Germany, 1996.

7. M. Germano, U. Piomelli, P. Moin, and W. Cabot, A dynamic subgrid-scale eddy viscosity model, Phys Fluids A 3 (1991), 1760-1765.

8. T. J. Hughes, L. Mazzei, and K. E. Jansen, Large eddy simulation and the variational multiscale method, Comput Visual Sci 3 (2000), 47-59.

9. P. Sagaut, Large eddy simulation for incompressible flows, Springer-Verlag, Berlin, Heidelberg, New York, 2001. 
10. V. John, W. J. Layton, and N. Sahin, Derivation and analysis of near wall models for channel and recirculating flows, Technical Report, University of Pittsburgh, 2002, submitted.

11. P. M. Gresho and R. L. Sani, Incompressible Flow and the Finite Element Method, Wiley, New York, 2000.

12. A. Leonard, Energy cascade in large eddy simulation of turbulent fluid flows, Adv Geophysics 18A (1974), 237-248.

13. R. A. Clark, J. H. Ferziger, and W. C. Reynolds, Evaluation of subgrid-scale models using an accurately simulated turbulent flow, J Fluid Mech 91 (1979), 1-16.

14. A. A. Aldama, Filtering techniques for turbulent flow simulation, Vol. 56 of Springer Lecture Notes in English, Springer, Berlin, 1990.

15. M. E. Cantekin, J. J. Westerink, and R. A. Luettich, Jr., Low and moderate Reynolds number transient flow simulations using space filtered Navier-Stokes equations, Num Meth Part Diff Equ 10 (1994), 491-524.

16. J. Heywood and R. Rannacher, Finite element approximation of the nonstationary Navier-Stokes problem i: Regularity of solutions and second order error estimates for spatial discretization, SIAM J Num Anal 19 (1982), 275-311.

17. J. Heywood and R. Rannacher, Finite element approximation of the nonstationary Navier-Stokes problem iii: Smoothing property and higher order estimates for spatial discretization, SIAM J Num Anal 25 (1988), 489-512.

18. W. J. Layton, Weak imposition of "no-slip" conditions in finite element methods, Comput Math Appl 38 (1999), 129-142.

19. A. Liakos, Weak imposition of boundary conditions in the Stokes and Navier-Stokes equation, $\mathrm{PhD}$ thesis, Univ. of Pittsburgh, 1999.

20. Q. Du and M. D. Gunzburger, Finite-element approximations of a Ladyzhenskaya model for stationary incompressible viscous flow, SIAM J Numer Anal 27 (1990), 1-19.

21. T. Iliescu, Genuinely nonlinear models for convection-dominated problems, Preprint ANL/MCSP857-1100, Argonne National Laboratory, 2000, submitted.

22. V. John and W. J. Layton, Analysis of numerical errors in large eddy simulation, SIAM J Numer Anal 2002, accepted for publication.

23. G. P. Galdi, An introduction to the mathematical theory of the Navier-Stokes equations, Vol. I: Linearized theory, Vol. 38 of Springer tracts in natural philosophy, Springer-Verlag, New York, 1994.

24. P. Coletti, A global existence theorem for large eddy simulation turbulence model, Math Models Meth Appl Sci 7 (1997), 579-591.

25. P. Coletti, Analytic and numerical results for $k-\epsilon$ and large eddy simulation turbulence models, $\mathrm{PhD}$ thesis, University of Trento, 1998.

26. L. G. Berselli, G. P. Galdi, T. Iliescu, and W. J. Layton, Mathematical analysis for the rational large eddy simulation model, Math Models Meth Appl Sci 12(8) (2002), 1-22.

27. O. A. Ladyzhenskaya, New equations for the description of motion of viscous incompressible fluids and solvability in the large of boundary value problems for them, Proc Steklov Inst Math 102 (1967), 95-118.

28. O. Axelsson and W. J. Layton, Defect correction methods for convection dominated convection diffusion methods, RAIRO Anal Numer 24 (1990), 423-455.

29. M. M. Vainberg, Variational methods and the method of monotone operators in the theory of nonlinear equations, Wiley, New York, 1973.

30. V. Girault and P. A. Raviart, Finite element approximation of the Navier-Stokes equations, SpringerVerlag, Berlin, Heidelberg, New York, 1979. 
31. M. Gunzburger, Finite element methods for viscous incompressible flows, Academic Press, Boston, 1989.

32. G. Minty, Monotone (nonlinear) operators in Hilbert space, Duke Math J 29 (1962), 341-346.

33. J. L. Lions, Quelques Méthodes de Résolution des Problèmes aux Limites Non Linéaires, Dunod, Gauthier-Villars, Paris, 1969.

34. G. Duvaut and J. L. Lions, Les Inéquations en Méchanique et Physique, Dunod, Paris, 1972.

35. M. Crouzeix and V. Thomée, The stability in $L^{p}$ and $W^{1, p}$ of the $L^{2}$-projection onto finite element function spaces, Math Comp 48 (1987), 521-523.

36. J. Xu, Theory of multilevel methods, PhD thesis, Cornell University, Ithaca, NY, 1989.

37. W. J. Layton, Analysis of a scale-similarity model of the motion of large eddies in turbulent flows, $\mathrm{J}$ Math Anal Appl 264 (2001), 546-559.

38. W. J. Layton and L. Tobiska, A two-level method with backtracking for the Navier-Stokes equations, SIAM J Numer Anal 35(5) (1998), 2035-2054.

39. M. O. Bristeau, R. Glowinski, and J. Periaux, Numerical methods for the Navier-Stokes equations: Applications to the simulation of compressible and incompressible viscous flows, Comput Phys Reports 6 (1987), 73-187.

40. P. Kloucek and F. S. Rys, Stability of the fractional step $\theta$-scheme for the nonstationary Navier-Stokes equations, SIAM Num Anal 31 (1994), 1312-1335.

41. S. Turek, Efficient solvers for incompressible flow problems: an algorithmic and computational approach, Vol. 6 of Lecture notes in computational science and engineering, Springer, 1999.

42. R. Rannacher, Finite element methods for the incompressible Navier-Stokes equations, G. P. Galdi, J. G. Heywood, and R. Rannacher, editors, Fundamental directions in mathematical fluid dynamics, Birkhäuser, 2000, pp. 191-293.

43. M. Fortin, Finite element solution of the Navier-Stokes equations, Acta Numerica (1993), 239-284.

44. V. John and G. Matthies, Higher order finite element discretizations in a benchmark problem for incompressible flows, Int J Num Meth Fluids 37 (2001), 885-901.

45. V. John, Higher order finite element methods and multigrid solvers in a benchmark problem for the 3D Navier-Stokes equations, Int J Num Meth Fluids 2002, accepted for publication.

46. Y. Saad, Iterative methods for sparse linear systems, PWS Publishing Company, 1996.

47. V. John and L. Tobiska, Smoothers in coupled multigrid methods for the parallel solution of the incompressible Navier-Stokes equations, Int J Num Meth Fluids 33 (2000), 453-473.

48. A. J. Chorin, Numerical solution for the Navier-Stokes equations, Math Comp 22 (1968), 745-762.

49. D. Tafti, Comparison of some upwind-biased high-order formulations with a second-order centraldifference scheme for time integration of the incompressible Navier-Stokes equations, Comp Fluids 25 (1996), 647-665.

50. V. John, Slip with friction and penetration with resistance boundary conditions for the Navier-Stokes equations-numerical tests and aspects of the implementation, J Comp Appl Math 2002, accepted for publication.

51. T. Iliescu, V. John, W. J. Layton, G. Matthies, and L. Tobiska, A numerical study of a class of les models, Int J Comput Fluid Dyn 2002, accepted for publication.

52. J. G. Heywood, R. Rannacher, and S. Turek, Artificial boundaries and flux and pressure conditions for the incompressible Navier-Stokes equations, Int J Numer Methods Fluids 22(5) (1996), 325-352. 